Review

\title{
The Potential of Introduction of Asian Vegetables in Europe
}

\author{
Jungha Hong and Nazim S. Gruda *(1) \\ Institute of Plant Sciences and Resource Conservation, Division of Horticultural Sciences, Faculty of Agriculture, \\ University of Bonn, 53121 Bonn, Germany; jungha.hong.miru@gmail.com \\ * Correspondence: ngruda@uni-bonn.de
}

Received: 1 May 2020; Accepted: 30 June 2020; Published: 3 July 2020

check for updates

\begin{abstract}
Increasing longevity, along with an aging population in Europe, has caused serious concerns about diet-related chronic diseases such as obesity, diabetes, cardiovascular diseases, and certain cancers. As recently noted during the coronavirus pandemic, regular exercise and a robust immune system complemented by adequate consumption of fruit and vegetables are recommended due to their known health benefits. Although the volume of fresh vegetable consumption in the EU is barely growing, demand for diversified, nutritious, and exotic vegetables has been increasing. Therefore, the European market for fresh Asian vegetables is expected to expand across the EU member states, and the introduction of new vegetables has enormous potential. We conducted this review to address the high number and wide range of Asian vegetable species with a commercial potential for introduction into the current European vegetable market. Many of them have not received any attention yet. Four Asian vegetables: (1) Korean ginseng sprout, (2) Korean cabbage, (3) Coastal hog fennel and (4) Japanese (Chinese or Korean) angelica tree, are further discussed. All of these vegetables possess several health benefits, are increasingly in demand, are easy to cultivate, and align with current trends of the European vegetable market, e.g., vegetables having a unique taste, higher value, are decorative and small. Introducing Asian vegetables will enhance the diversity of nutritious horticultural products in Europe, associated with all their respective consumption benefits. Future research on the Asian vegetable market within Europe is needed. In addition, experimental studies of Asian vegetables under practical conditions for their production in different European environments are required. Economic, social, and ecological aspects also ought to be considered.
\end{abstract}

Keywords: health; aging population; consumption of fruit and vegetables; diversification; market trend; Korean ginseng sprout; Ssamchoo; Peucedanum japonicum; Aralia elata (Miq.) Seem

\section{Introduction}

Health and well-being are important factors of a modern lifestyle. The health conditions of individuals and households have economic significance for both developing and developed countries, including the EU member states [1]. Additionally, an aging population is considered a significant challenge in the coming decades for the European Union [2]. Worldwide chronic diseases are increasing as leading causes of death every year, with scientific evidence linking these chronic diseases to diet [3]. These diet-related chronic diseases include obesity, diabetes, cardiovascular diseases (CVDs), and certain cancers [3-6].

Obesity is associated with various health problems including CVD, type 2 diabetes mellitus, musculoskeletal disorders such as osteoarthritis, work disability and respiratory disorders [7]. According to a 2016 report from the Organization for Economic Co-operation and Development/ European Union (OECD/EU [8], 16\% of adults in the EU member states were obese in 2004, an increase 
from $11 \%$ in 2000 . Moreover, overweight or obese children have a higher risk of poor health in adulthood [9].

Diabetes causes a higher risk of developing cardiovascular diseases such as heart attack and stroke [8]. Across EU countries, $7 \%$ of adults reported having diabetes in a self-reported interview survey in 2014. The rate of diabetes is associated with the level of education and age [8].

CVDs are the leading cause of mortality in nearly all EU countries [8]. CVDs caused almost $40 \%$ of all deaths in 2013 [8]. CVDs include a wide range of diseases, such as ischemic heart diseases (e.g., heart attack) and cerebrovascular diseases (e.g., stroke) [8].

Cancer is the second leading cause of mortality after cardiovascular diseases in EU countries [8]. $26 \%$ of all deaths were caused by cancer in 2013 [8]. Cancer mortality rates for men are higher than for women in all EU countries [8]. Considered risk factors for colorectal cancer are age, a diet high in fat, and genetic background [8].

According to WHO, the COVID-19 pandemic is caused by a novel coronavirus [10,11]. A study in China reported that approximately $50 \%$ of patients confirmed with COVID-19 had underlying chronic diseases such as CVDs (40\%) and diabetes [12]. Another retrospective analysis in Wuhan showed that obesity was commonly observed in critical patients and death from COVID-19 [13]. According to this study, $88.24 \%$ of patients with a BMI (body mass index) over $25 \mathrm{~kg} / \mathrm{m}^{2}$ were in the non-survivor group [13]. Based on current evidence, COVID-19 is considered to be highly associated with a compromised immune system of individuals that are affected by age, gender and comorbidities (CVDs, diabetes, cancer) [14]. Understanding the significant correlation among diet, nutrition, infection, and immunity [15], a diversified, balanced, and healthy diet with macro-, micro- and phytonutrients, mainly found in vegetables, may promote healthy immune responses for prevention of chronic diseases [16].

European consumers are growing more interested in diversified, nutritious, and exotic vegetables. The cultivation and consumption of Asian vegetables are increasing in Europe. For instance, Chinese cabbage (Brassica pekinensis) [17-20] and pak choi (Brassica chinensis) [17,18,20,21], which were unknown some years ago, are the two most popular Asian vegetables in Europe. These leafy vegetables originating from China [22] are essential in northeastern Asia. However, as they started to be commercially cultivated in Europe, bolting was a significant concern for European growers [23]. Bolting is induced by vernalization which is a process of accelerated flowering by exposure to prolonged cold [24] that results in elongation of the flower stalk [25]. Vernalization lets the plants bloom and produce seeds instead of leafy heads [26], which then lead to low yield and quality loss of pak choi [27] and Chinese cabbage. As a result, many research studies of cultivation have shown how to control bolting and early flowering of Chinese cabbage and pak choi.

Mizuna [17,20], a cultivar of Brassica rapa var. niposinica, and water spinach (Ipomoea aquatica) $[17,20,21]$ are other Asian vegetables introduced to Europe. They both are less well known than Chinese cabbage and pak choi in Europe. Mizuna is a Japanese vegetable [28] that is mostly cultivated in open fields but also inside greenhouses in Italy $[29,30]$. There is also a concern about bolting for commercial production of mizuna [28]. In contrast, to our knowledge, water spinach is consumed but is not commercially cultivated in Europe.

Currently, shiso (Perilla frutescens var. crispa), which is a Japanese herb, is cultivated in greenhouses in Germany [31]. Choy sum (Brassica rapa var. parachinensis), kai choi (Brassica juncea) [17-21], edamame (Glycine hispida), Japanese murasaki sweet potato (Ipomoea batatas), Okinawa sweet potato (Ipomoea batatas cv. 'Ayamurasaki') are produced in Portugal [32].

Carotenoids, anthocyanins, flavonoids, and other phenolic compounds in vegetables may prevent and counter many common diseases such as high blood pressure, diabetes, cancer, vision loss, heart disease, and several intestinal disorders. The link between fruit and vegetable consumption and a lower risk of mortality has been increasing [33-35]. Cardiovascular mortality may notably decrease with high consumption of fruit and vegetables [36]. It was also noticed that not only the elderly and chronic disease sufferers, but also smokers and alcohol drinkers were in the high-risk patient group 
during the Covid-19 pandemic. Thus, eating vegetables is suggested together with sporting activities to boost the immune system [16,37].

To our best knowledge, no published literature to date provides a comprehensive review of Asian vegetables that have the potential to be introduced in Europe. The objective of this paper is to contribute to an increasing diversity of vegetable consumption in Europe.

\section{Vegetable Consumption in Europe}

According to Shahbaz et al. [38], vegetables provide a variety of health benefits. They are generally low in fat and calories yet rich in vitamins and dietary fibers. Moreover, they present a valuable source of mineral nutrients, essential amino acids, antioxidants and phytochemicals such as carotenoids, anthocyanins, flavonoids, and other phenolic compounds.

Besides the nutritional attributes, vegetables also provide a diverse range of taste, aroma, texture, and color, which significantly increase the variety in food and satisfy a myriad of personal preferences [39]. Although increased daily vegetable intake has been recommended for health benefits, and the EU has made efforts to advocate it, European vegetable consumption remains below the recommended level [40-42]

\section{1. '5-A-day' Campaign of Daily Vegetable E Fruits Consumption}

WHO recommended daily consumption of a minimum $400 \mathrm{~g}$ of fruits and vegetables to prevent chronic diseases in 1990 [43]. After that, the '5-a-day' fruit and vegetable campaign was launched in several European countries such as the UK, Germany, and France [43]. The '5-a-day' fruit and vegetable campaign recommended at least five portions of fruits and vegetables a day, excluding potatoes and other starchy tubers [44]. Indeed, evidence provided by a meta-analysis showed that consumption of vegetables and fruit-up to five servings a day-was associated with a lower risk of all causes of mortality, especially cardiovascular mortality [36]. In addition, the consumption of similar amounts of vegetables appears to be significantly healthier than that of fruits $[43,45]$ because the fructose found in fruit can be a major factor of obesity [46-48].

\subsection{EU Policy to Promote Vegetable \& Fruits Consumption}

The EU has made efforts to promote a well-balanced diet to prevent chronic diseases. One of the policy objectives of the EU is the promotion of fruit and vegetable consumption, especially in schools and workplaces [49]. The European School Fruit Scheme, which supplies fresh fruit and vegetables to children together with educational measures, was introduced in the schools of $24 \mathrm{EU}$ countries in 2009-2010 [8]. Since then, the European School Fruit Scheme has been positively showing a significant increase in fruit and vegetable intake frequency by children [8]. Caton et al. [40], reported the importance of learning to eat vegetables at an early age. The European Commission is monitoring progress in the consumption of fruit and vegetables to maintain a balance against a worsening trend of poor diets [8,50]. The WHO European Food and Nutrition Action Plan 2015-20 was designed to reduce the burden of preventable diet-related NCDs (Noncommunicable diseases) and obesity [8].

\subsection{Daily Vegetable (E Fruits) Consumption of Young (Girls $\mathcal{E}$ Boys) and Adults in the EU}

According to OECD/EU [8], over one in three girls and one in four boys ate vegetables daily in EU countries in 2013-14. The average daily vegetable consumption of adults was $51 \%$ in $28 \mathrm{EU}$ countries in 2014. More women eat vegetables daily than men and older adults eat more fruits and vegetables daily than younger people do. About $12 \%$ of adults in $28 \mathrm{EU}$ countries have reported a daily consumption of at least five fruits and vegetables. People with higher education levels tend to eat fruits and vegetables more often than those with lower education levels. 


\subsection{Daily Vegetable Consumption of European Countries-A Systematic Comparison with Asian Countries}

According to the Food and Agriculture Organization of the United Nations (FAO) (1999) [51], about 75 percent of the world's food is generated from only 12 plants and five animal species. A recent study by international scientists in collaboration with the FAO identified a total of 1097 vegetable species worldwide [52] of which 392 vegetable crops are cultivated and consumed [39]. However, even in the current globalization era, there are significant differences between countries. More than 200 species are known to be produced in Asian countries whereas the maximum number of vegetable species is only 60 coming from 16 plant families in Germany. Considering the fact that approximately $81 \%$ of the consumption/capita/year is concentrated on 23 vegetable species, it is clear that the diversity of vegetables in Germany is relatively weak [53]. This situation is reflected in the average rate of daily vegetable consumption.

Data sourced by OECD Health Statistics (2017) [54] showed that the average rate of daily vegetable consumption among adults in OECD 32 member countries is $59.8 \%$. The data of European countries are all lower than of those other countries including Korea (99.1\%), Australia (99.0\%), New Zealand (95.3\%) and the United States (92.4\%) (unfortunately, no data for China were recorded) [54].

China was the world's largest fresh vegetable producer in 2017 [55] and was the world's biggest vegetable exporter in 2018 [56]. The FAO and World Bank reported in 2017 [57] that China was likely to consume the most vegetables per capita (1033 g/day). In other Asian countries, South Korea consumed $540.14 \mathrm{~g}$, Vietnam $467.25 \mathrm{~g}$, Japan $249.61 \mathrm{~g}$, and Thailand $109.3 \mathrm{~g}$ per day [57].

Among the Mediterranean countries, Croatia consumed the most vegetables per capita (699.03 g/day), followed by Portugal (418.62 g/day), Greece (410.84 g/day), and Spain (401.52 g/day) [57]. In Northwestern Europe, Belgium consumed the most vegetables ( $379.46 \mathrm{~g} /$ day), followed by Denmark (276.44 g/day), France (264.85 g/day) and Germany (261.29 g/day) [57].

Despite the current recommendations and the apparent health benefits, vegetable consumption in European countries is below the recommended level [40-42]. For instance, in Germany, the consumption per capita has increased from $186.3 \mathrm{~g}$ to $261.38 \mathrm{~g}$ in the past 30 years [53,57]. However, consumption has stagnated at approximately $94-95 \mathrm{~kg} / \mathrm{capita} / \mathrm{year}$ for many years now. The recent numbers include the comprehensive campaigns that were previously mentioned, including the $5 \mathrm{X}$ a day campaigns, activities for pre- and school-children, as well as information targeting adults concerning health issues.

Therefore, one possible way to increase the consumption of vegetables in EU countries could be diversification. Consumers are looking for new products, especially in the winter with reduced varieties available, which could be an excellent opportunity for new vegetables coming from Asian countries.

\section{The Potential for the Introduction of New Asian Vegetables in Europe and the Market Trends}

The consumption of fresh vegetables in the EU is barely increasing, and the European vegetable market is highly competitive. However, the current demand for healthy, high-quality and attractive exotic vegetables is growing steadily. Therefore, the market for fresh Asian vegetables has the potential to expand across the EU member states. But, how can we define Asian vegetables?

The meaning of "Asian vegetables" varies and the term is often used ambiguously as "Oriental vegetables" in literature. Asian vegetables can be defined as vegetables that have originated, are cultivated or are commonly consumed in Northeast Asia (China, Korea, and Japan) or in Southeast Asia (Thailand, Indonesia, Vietnam, the Philippines, and Malaysia). In this study, we will use the term Asian vegetables to mean the vegetables from both regions.

A list of Asian vegetables is provided in Tables 1-6. In the list, some well-known vegetables that can be found in other countries are not included, such as onions (Allium cepa), potatoes (Solanum tuberosum), sweet potatoes (Ipomoea batatas), tomatoes (Solanum lycopersicum), and okra (Abelmoschus esculentus) even though they are commonly used in Asian cuisines. However, we still included these vegetables if they are unique from their western counterparts, such as Asian green onions (Allium fistulosum), Japanese pumpkin (Cucurbita maxima), and Korean zucchini (Cucurbita moschata). Native Asian vegetables such as soybeans and Chinese cabbage are also included. 
Table 1. Sprout vegetables.

\begin{tabular}{|c|c|c|c|c|c|c|c|c|c|}
\hline Vegetable & Scientific Names & Used Parts & Culinary Uses & Reference & Vegetable & Scientific Names & Used Parts & Culinary Uses & References \\
\hline $\begin{array}{l}\text { Mung bean sprouts/Mungo bean } \\
\text { sprouts/Small bean sprouts }\end{array}$ & $\begin{array}{l}\text { Phaseolus aureus/Vigna } \\
\text { radiata }\end{array}$ & sprouts & $\begin{array}{l}\text { eaten raw, blanched, } \\
\text { stir-fried }\end{array}$ & {$[17,18,21]$} & Soybean sprouts & Glycine hispida/G. max & sprouts & blanched, boiled & {$[17,18,21]$} \\
\hline
\end{tabular}

Table 2. Tree vegetables.

\begin{tabular}{|c|c|c|c|c|c|c|c|c|c|}
\hline Vegetable & Scientific Names & Used Parts & Culinary Uses & Reference & Vegetable & Scientific Names & Used Parts & Culinary Uses & References \\
\hline $\begin{array}{l}\text { Aralia sprout/Dureup/Fatsia } \\
\text { sprout/Korean angelica tree }\end{array}$ & $\begin{array}{l}\text { Aralia elata (Miq.) Seem./Aralia } \\
\text { elata var. elata }\end{array}$ & young shoots & blanched, pickled & {$[18,19,58]$} & $\begin{array}{l}\text { Alangium/Trilobed-leaf } \\
\text { alangium }\end{array}$ & $\begin{array}{l}\text { Alangium platanifolium } \\
\text { var. trilobum (Miq.) Ohwi }\end{array}$ & young leaves & $\begin{array}{l}\text { eaten raw young leaves, } \\
\text { blanched, pickled }\end{array}$ & {$[18,58]$} \\
\hline Bamboo shoot & $\begin{array}{l}\text { Bambusa spp./Bambusa } \\
\text { sp./Dendrocalamus } \\
\text { sp./Phyllostachys sp. }\end{array}$ & young shoots & $\begin{array}{l}\text { blanched, boiled, pickled, } \\
\text { stir-fried }\end{array}$ & {$[17,18,21]$} & Ginkgo nut & Ginkgo biloba & seeds & cooked, grilled & [18] \\
\hline Burning bush spindle tree & $\begin{array}{l}\text { Euonymus alatus (Thunb.) } \\
\text { Siebold }\end{array}$ & young shoots & blanched & [18] & $\begin{array}{l}\text { Blue Japanese oak } \\
\text { acorn/Ring-cup oak acorn }\end{array}$ & Quercus glauca Thunb. & acorns & $\begin{array}{l}\text { its starch is cooked and } \\
\text { made jelly }\end{array}$ & {$[18,58]$} \\
\hline Chinese cedar & Cedrela sinensis Juss. & young shoots & $\begin{array}{l}\text { blanched, pickled, deep } \\
\text { fried }\end{array}$ & {$[18,19]$} & Konara oak acorn & Quercus serrata Murray & acorns & $\begin{array}{l}\text { its starch is cooked and } \\
\text { made jelly }\end{array}$ & {$[18,58]$} \\
\hline $\begin{array}{l}\text { Devil's } \\
\text { bush/Siberian-ginseng }\end{array}$ & Eleutherococcus senticosus & young shoots & blanched & {$[18,58]$} & $\begin{array}{l}\text { Sansho pepper/Sichuan } \\
\text { pepper }\end{array}$ & Zanthoxylum sp. & husks of seeds & $\begin{array}{l}\text { adding in stews, foods, } \\
\text { oiled }\end{array}$ & [20] \\
\hline Matrimony-vine & Lycium chinense & young shoots & blanched young leaf buds & {$[17,18]$} & $\begin{array}{l}\text { East Asian arrow } \\
\text { root/Kudzu }\end{array}$ & $\begin{array}{l}\text { Dolichos japonica/Pueraraia } \\
\text { hirsute/P. lobata (Willd.) } \\
\text { OOhi/P. thunbergiana) }\end{array}$ & roots & $\begin{array}{l}\text { eaten raw, made juice, } \\
\text { dried and boiled tea, } \\
\text { made jelly, powdered }\end{array}$ & {$[17,18,58]$} \\
\hline Sayur manis/weet leaf bush & Sauropus androgynus & $\begin{array}{l}\text { shoot tips, } \\
\text { young leaves }\end{array}$ & $\begin{array}{l}\text { steamed, grilled, cooked in } \\
\text { soups, stir-fried }\end{array}$ & [21] & & & & & \\
\hline
\end{tabular}

Table 3. Leaf and Stem Vegetables.

\begin{tabular}{|c|c|c|c|c|c|c|c|c|c|}
\hline $\begin{array}{l}\text { Vegetable } \\
\end{array}$ & Scientific Names & Used Parts & Culinary Uses & Reference & Vegetable & Scientific Names & Used Parts & Culinary Uses & References \\
\hline Asian goldenrod & $\begin{array}{l}\text { Solidago virgaurea subsp. asiatica } \\
\text { Kitam. ex H. Hara }\end{array}$ & young shoots & blanched & {$[18,19,58]$} & Komatsuna & $\begin{array}{l}\text { Brassica rapa var. } \\
\text { komatsuna/var. perviridis }\end{array}$ & leaves, stalks & $\begin{array}{l}\text { eaten raw, stir-fried, } \\
\text { boiled, pickled, cooked in } \\
\text { soups }\end{array}$ & [20] \\
\hline Asian royal fern/Royal fern & Osmunda japonica Thunb. & young shoots & boiled, stir-fried & {$[18,19,58]$} & $\begin{array}{l}\text { Korean } \\
\text { cabbage/Ssamchoo }\end{array}$ & $\begin{array}{l}\text { Brassica lee ssp. namai cv. } \\
\text { Ssamchoo }\end{array}$ & leaves, stalks & eaten raw & [18] \\
\hline $\begin{array}{l}\text { Bracken/Eastern } \\
\text { brakenfern/Tender fern } \\
\text { fronds }\end{array}$ & $\begin{array}{l}\text { Pteridium aquilinum var. } \\
\text { latiusculum (Desv.) Underw. ex } \\
\text { A.Heller }\end{array}$ & young shoots & boiled, stir-fried & {$[17,19,58]$} & Mibuna greens & $\begin{array}{l}\text { Brassica rapa var. } \\
\text { japonica/var. nipposinica }\end{array}$ & leaves, stalks & eaten raw & [20] \\
\hline Edible aster/Rough aster & Aster scaber Thunberg & young shoots & eaten raw & {$[18,19,58]$} & $\begin{array}{l}\text { Mizuna } \\
\text { greens/Potherb-mustard } \\
\text { cabbage }\end{array}$ & $\begin{array}{l}\text { Brassica rapa var. } \\
\text { japonica/var. nipposinica }\end{array}$ & leaves, stalks & $\begin{array}{l}\text { eaten raw, stir-fried, } \\
\text { cooked in soups }\end{array}$ & {$[17,20]$} \\
\hline $\begin{array}{l}\text { Japanese } \\
\text { spikenard/Manchurian } \\
\text { spikenard/Mountain } \\
\text { asparagus/Udo }\end{array}$ & $\begin{array}{l}\text { Aralia cordata var. continentalis } \\
\text { (Kitag.) Y.C.Chu }\end{array}$ & young shoots & $\begin{array}{l}\text { blanched, deep fried, } \\
\text { cooked as pancakes }\end{array}$ & {$[18,20]$} & Turnip greens & Brassica rapa & leaves, stalks & boiled, stir-fried, salted & {$[17,18]$} \\
\hline Pea shoots & Pisum sativum & young shoots & stir-fried & [20] & $\begin{array}{l}\text { Chinese taro/Japanese } \\
\text { taro/Taro }\end{array}$ & Colocasia esculenta & stalks & boiled, stir-fried & {$[17,18]$} \\
\hline
\end{tabular}


Table 3. Cont.

\begin{tabular}{|c|c|c|c|c|c|c|c|c|c|}
\hline Vegetable & Scientific Names & Used Parts & Culinary Uses & Reference & Vegetable & Scientific Names & Used Parts & Culinary Uses & References \\
\hline $\begin{array}{l}\text { Tartarian aster/Tatarinow's } \\
\text { aster }\end{array}$ & Aster tataricus L. f. & young shoots & blanched & {$[18,19,58]$} & $\begin{array}{l}\text { Citronella grass/Lemon } \\
\text { grass }\end{array}$ & Cymbopogon citratus & stalks & cooked in curries, soups & [20] \\
\hline Water shield & Brasenia schreberi J.F.Gmelin & young shoots & $\begin{array}{l}\text { eaten raw, cooked in soups, } \\
\text { dried, grounded }\end{array}$ & {$[20,58]$} & Sweet potato & Ipomoea batatas & stalks & $\begin{array}{l}\text { blanched, stir-fried, } \\
\text { salted }\end{array}$ & [17] \\
\hline Hot pepper & Capsicum аппиит & $\begin{array}{l}\text { young shoots, } \\
\text { leaves }\end{array}$ & $\begin{array}{l}\text { Blanched, cooked in soups } \\
\text { and in pancakes }\end{array}$ & [18] & $\begin{array}{l}\text { Amaranthus/Chinese } \\
\text { spinach/Edible } \\
\text { amaranth/Pig weed }\end{array}$ & $\begin{array}{l}\text { Amaranthus gangeticus/A. } \\
\text { mangostanus L./A. tricolor }\end{array}$ & $\begin{array}{l}\text { young leaves, } \\
\text { stems }\end{array}$ & $\begin{array}{l}\text { blanched, stir-fried, } \\
\text { cooked in soups }\end{array}$ & {$[17-21,58]$} \\
\hline $\begin{array}{l}\text { Komarov's Russian } \\
\text { thistle/Okahijiki }\end{array}$ & Salsola komarovii Iljin & $\begin{array}{l}\text { young shoots, } \\
\text { leaves }\end{array}$ & $\begin{array}{l}\text { eaten raw, blanched, } \\
\text { steamed }\end{array}$ & {$[20,58]$} & Ashitaba & Angelica keiskei & $\begin{array}{l}\text { young leaves, } \\
\text { stems }\end{array}$ & $\begin{array}{l}\text { eaten raw, blanched, } \\
\text { cooked in pancakes, } \\
\text { pickled }\end{array}$ & {$[18,19]$} \\
\hline Korean-mint & Agastache rugosa & $\begin{array}{l}\text { young shoots, } \\
\text { leaves }\end{array}$ & $\begin{array}{l}\text { eaten raw young leaves, } \\
\text { blanched, cooked in soups, } \\
\text { as pancakes }\end{array}$ & [18] & Coastal hog fennel & $\begin{array}{l}\text { Peucedanum japonicum } \\
\text { Thunb. }\end{array}$ & $\begin{array}{l}\text { young leaves, } \\
\text { stems }\end{array}$ & $\begin{array}{l}\text { eaten raw, pickled, } \\
\text { blanched, deep fried }\end{array}$ & {$[18,19,58]$} \\
\hline $\begin{array}{l}\text { Okinawan spinach/Velvet } \\
\text { plant }\end{array}$ & Gynura bicolor & $\begin{array}{l}\text { young shoots, } \\
\text { leaves }\end{array}$ & $\begin{array}{l}\text { eaten raw, blanched, } \\
\text { stir-fried, deep-fried, } \\
\text { cooked in soups, stews }\end{array}$ & [20] & Curled mallow/Mallows & $\begin{array}{l}\text { Malva verticillata/M. } \\
\text { verticillata var. crispa } \mathrm{L} \text {. }\end{array}$ & $\begin{array}{l}\text { young leaves, } \\
\text { stems }\end{array}$ & $\begin{array}{l}\text { cooked in soups, } \\
\text { stir-fried }\end{array}$ & [18-20] \\
\hline Stringy stonecrop & Sedum sarmentosum & $\begin{array}{l}\text { young shoots, } \\
\text { leaves }\end{array}$ & eaten raw & {$[18,19]$} & East Asian hogweed & $\begin{array}{l}\text { Heracleum moellendorffii } \\
\text { Hance }\end{array}$ & $\begin{array}{l}\text { young leaves, } \\
\text { stems }\end{array}$ & $\begin{array}{l}\text { eaten raw, blanched, } \\
\text { pickled, stir-fried }\end{array}$ & {$[18,19,58]$} \\
\hline $\begin{array}{l}\text { Ceylon spinach/Malabar } \\
\text { nightshade/Tsurumurasaki }\end{array}$ & Basella alba/B. cordifolia B. rubra/ & shoots, leaves & stir-fried, cooked in soups & {$[17,20,21]$} & Field aster & $\begin{array}{l}\text { Aster yomena (Kitam.) } \\
\text { Honda }\end{array}$ & $\begin{array}{l}\text { young leaves, } \\
\text { stems }\end{array}$ & $\begin{array}{l}\text { blanched, stir-fried, } \\
\text { cooked in soups, deep } \\
\text { fried }\end{array}$ & {$[18,58]$} \\
\hline Castor & Ricinus communis $\mathrm{L}$. & young leaves & blanched & [18] & Gondre/Korean thistle & $\begin{array}{l}\text { Cirsium setidens (Dunn) } \\
\text { Nakai }\end{array}$ & $\begin{array}{l}\text { young leaves, } \\
\text { stems }\end{array}$ & $\begin{array}{l}\text { cooked in soups, pickled, } \\
\text { dried leaves are cooked } \\
\text { as side dishes }\end{array}$ & {$[18,19,58]$} \\
\hline $\begin{array}{l}\text { Chinese chives/Flat } \\
\text { chives/Garlic chives }\end{array}$ & $\begin{array}{l}\text { Allium senescens/A. senescens var. } \\
\text { minor/A. thunbergii/A. tuberosum }\end{array}$ & leaves & $\begin{array}{l}\text { eaten raw, stir-fried, } \\
\text { pickled, cooked in } \\
\text { pancakes }\end{array}$ & [18-21] & Japanese angelica & Ligusticum acutilobum & $\begin{array}{l}\text { young leaves, } \\
\text { stems }\end{array}$ & $\begin{array}{l}\text { eaten raw, blanched, } \\
\text { pickled }\end{array}$ & [18] \\
\hline Perilla & Perilla frutescens & leaves & $\begin{array}{l}\text { eaten raw, pickled, } \\
\text { stir-fried }\end{array}$ & {$[17,18,20]$} & $\begin{array}{l}\text { Japanese } \\
\text { atractylodes/Ovate-leaf } \\
\text { atractylodes }\end{array}$ & $\begin{array}{l}\text { Atractylodes japonica/A. } \\
\text { ovata (Thunb.) DC. }\end{array}$ & $\begin{array}{l}\text { young leaves, } \\
\text { stems }\end{array}$ & $\begin{array}{l}\text { eaten raw, blanched, } \\
\text { cooked in pancakes }\end{array}$ & {$[18,58]$} \\
\hline Soybean & Glycine max (L.) Merr. & leaves & blanched, pickled, salted & [18] & $\begin{array}{l}\text { Lesser solomon's } \\
\text { seal/Solomon's seal }\end{array}$ & $\begin{array}{l}\text { Polygonatum odoratum var. } \\
\text { pluriflorum (Miq.) Ohwi }\end{array}$ & $\begin{array}{l}\text { young leaves, } \\
\text { stems }\end{array}$ & pickled & {$[18,58]$} \\
\hline Asian plantain & Plantago asiatica $\mathrm{L}$. & $\begin{array}{l}\text { young leaves, } \\
\text { stalks }\end{array}$ & $\begin{array}{l}\text { blanched, cooked in soups, } \\
\text { stir-fried }\end{array}$ & {$[18,58]$} & $\begin{array}{l}\text { Riverside } \\
\text { wormwood/Selenge } \\
\text { wormwood }\end{array}$ & $\begin{array}{l}\text { Artemisia selengensis } \\
\text { Turcz. ex Besser }\end{array}$ & $\begin{array}{l}\text { young leaves, } \\
\text { stems }\end{array}$ & blanched & {$[18,58]$} \\
\hline $\begin{array}{l}\text { Butterbur/Giant } \\
\text { butterbur/Sweet coltsfoot/ }\end{array}$ & $\begin{array}{l}\text { Petasites japonicus (Siebold \& } \\
\text { Zucc.) Maxim. }\end{array}$ & $\begin{array}{l}\text { young leaves, } \\
\text { stalks }\end{array}$ & blanched, pickled & {$[17-20,58]$} & $\begin{array}{l}\text { Swamp cabbage/Water } \\
\text { convolvulus/Water } \\
\text { spinach }\end{array}$ & Ipomoea aquatica/I. reptans & $\begin{array}{l}\text { young leaves, } \\
\text { stems }\end{array}$ & $\begin{array}{l}\text { Eaten raw, blanched, } \\
\text { boiled, stir-fried }\end{array}$ & {$[17,20,21]$} \\
\hline $\begin{array}{l}\text { Chamnamul/Short-fruit } \\
\text { pimpinella }\end{array}$ & $\begin{array}{l}\text { Pimpinella brachycarpa (Kom.) } \\
\text { Nakai }\end{array}$ & $\begin{array}{l}\text { young leaves, } \\
\text { stalks }\end{array}$ & blanched & {$[18,19,58]$} & Three-leaf ladybell & $\begin{array}{l}\text { Adenophora triphylla var. } \\
\text { japonica (Regel) H. Hara }\end{array}$ & $\begin{array}{l}\text { young leaves, } \\
\text { stems }\end{array}$ & eaten raw, blanched & {$[18,58]$} \\
\hline Deltoid synurus & Synurus deltoides (Aiton) Nakai & $\begin{array}{l}\text { young leaves, } \\
\text { stalks }\end{array}$ & $\begin{array}{l}\text { blanched, cooked in rice } \\
\text { cakes }\end{array}$ & {$[18,19,58]$} & Ulleungdo aster & Aster glehnii F.Schmidt & $\begin{array}{l}\text { young leaves, } \\
\text { stems }\end{array}$ & blanched, deep fried & {$[18,58]$} \\
\hline
\end{tabular}


Table 3. Cont

\begin{tabular}{|c|c|c|c|c|c|c|c|c|c|}
\hline Vegetable & Scientific Names & Used Parts & Culinary Uses & Reference & $\begin{array}{l}\text { Vegetable } \\
\text {. }\end{array}$ & Scientific Names & Used Parts & Culinary Uses & References \\
\hline Fischer's ragwort/Gomchwi & Ligularia fischeri (Ledeb.) Turcz. & $\begin{array}{l}\text { young leaves, } \\
\text { stalks }\end{array}$ & $\begin{array}{l}\text { eaten raw, cooked in soups, } \\
\text { picked, blanched }\end{array}$ & {$[18,19,58]$} & $\begin{array}{l}\text { Water celery/Water } \\
\text { dropwort/Water parsley }\end{array}$ & $\begin{array}{l}\text { Oenanthe javanica/O. } \\
\text { stoloniferal }\end{array}$ & $\begin{array}{l}\text { young leaves, } \\
\text { stems }\end{array}$ & $\begin{array}{l}\text { eaten raw, blanched, } \\
\text { cooked in soups, as } \\
\text { pancake }\end{array}$ & {$[17-20]$} \\
\hline Honewort/Mitsuba & Cryptotaenia canadensis & $\begin{array}{l}\text { young leaves, } \\
\text { stalks }\end{array}$ & $\begin{array}{l}\text { eaten raw, stir-fried, deep } \\
\text { fired, cooked in soups }\end{array}$ & [17] & $\begin{array}{l}\text { Chinese broccoli/Chinese } \\
\text { kale }\end{array}$ & $\begin{array}{l}\text { Brassica oleracea var. } \\
\text { alboglabra }\end{array}$ & leaves, stems & boiled, steamed, stir-fried & {$[17,20,21]$} \\
\hline Korean bellflower & Campanula takesimana Nakai & $\begin{array}{l}\text { young leaves, } \\
\text { stalks }\end{array}$ & blanched & {$[18,19,58]$} & $\begin{array}{l}\text { Chrysanthemum/Chrysanth } \\
\text { greens/Crown } \\
\text { daisy/Garland } \\
\text { chrysanthemum }\end{array}$ & $\begin{array}{l}\text { nemum } \\
\text { Chrysanthemum } \\
\text { coronarium }\end{array}$ & leaves, stems & $\begin{array}{l}\text { eaten raw, cooked in } \\
\text { stews, blanched }\end{array}$ & {$[17-21]$} \\
\hline $\begin{array}{l}\text { Narrow spiked } \\
\text { Ligularia/Narrow-head } \\
\text { ragwort }\end{array}$ & $\begin{array}{l}\text { Ligularia stenocephala (Maxim.) } \\
\text { Matsum. \& Koidz. }\end{array}$ & $\begin{array}{l}\text { young leaves, } \\
\text { stalks }\end{array}$ & $\begin{array}{l}\text { eaten raw, cooked in soups, } \\
\text { picked, blanched }\end{array}$ & {$[18,19,58]$} & Stem lettuce & $\begin{array}{l}\text { Lactuca sativa var. } \\
\text { augustana/var. asparagina }\end{array}$ & leaves, stems & blanched, stir-fried & [20] \\
\hline Pumpkin & Cucurbita moschata & $\begin{array}{l}\text { young leaves, } \\
\text { stalks }\end{array}$ & steamed & [18] & $\begin{array}{l}\text { Manchurian wild } \\
\text { rice/Water bamboo/Wild } \\
\text { rice }\end{array}$ & $\begin{array}{l}\text { Zizania latifolia (Griseb.) } \\
\text { Turcz. ex Stapf }\end{array}$ & $\begin{array}{l}\text { swollen stem } \\
\text { bases }\end{array}$ & eaten raw, stir-fried & {$[20,58]$} \\
\hline Rape & Brassica napus $\mathrm{L}$. & $\begin{array}{l}\text { young leaves, } \\
\text { stalks }\end{array}$ & blanched, cooked in soups & {$[18,19]$} & $\begin{array}{l}\text { Green onion/Japanese } \\
\text { bunching onions/Oriental } \\
\text { bunching } \\
\text { onion/Scallions/Shallots/Spri } \\
\text { onion/Welsh onions }\end{array}$ & $\begin{array}{l}\text { Allium fistulosum } \\
\text { ring }\end{array}$ & leaves, bulbs & $\begin{array}{l}\text { eaten raw, stir-fried, } \\
\text { salted, cooked in soups, } \\
\text { pancakes }\end{array}$ & {$[17-21]$} \\
\hline Salt sandspurry & Spergularia marina (L.) & $\begin{array}{l}\text { young leaves, } \\
\text { stalks }\end{array}$ & $\begin{array}{l}\text { eaten raw, blanched, } \\
\text { cooked in pancakes }\end{array}$ & {$[18]$} & $\begin{array}{l}\text { Korean wild chive Wild } \\
\text { garlic }\end{array}$ & $\begin{array}{l}\text { Allium monanthum } \\
\text { Maxim. }\end{array}$ & leaves, bulbs & $\begin{array}{l}\text { eaten raw cooked in } \\
\text { soups, pickled }\end{array}$ & {$[18,19,58]$} \\
\hline $\begin{array}{l}\text { Bok choy/Chinese } \\
\text { cabbage/Pak choi/White } \\
\text { cabbage/White-mustard } \\
\text { cabbage }\end{array}$ & $\begin{array}{l}\text { Brassica campestris var. } \\
\text { chinensis/B. rapa var. chinensis }\end{array}$ & leaves, stalks & $\begin{array}{l}\text { eaten raw, blanched, } \\
\text { stir-fried }\end{array}$ & {$[17,18,20,21]$} & Korean youngia & Youngia sonchifolia & $\begin{array}{l}\text { young leaves, } \\
\text { roots }\end{array}$ & salted & {$[18,19]$} \\
\hline Chinese cabbage & Brassica pekinensis/B. pe-tsai & leaves, stalks & $\begin{array}{l}\text { eaten raw, salted, cooked in } \\
\text { soups, as pancakes, } \\
\text { pickled, stir-fried }\end{array}$ & [17-20] & Shepherd's purse & Capsella bursa-pastoris & $\begin{array}{l}\text { young leaves, } \\
\text { roots }\end{array}$ & $\begin{array}{l}\text { blanched, cooked in } \\
\text { soups }\end{array}$ & [18-20] \\
\hline Chinese celery & Apium graveolens & leaves, stalks & $\begin{array}{l}\text { eaten raw, stir-fired, cooked } \\
\text { in soups, steamed, pickled }\end{array}$ & {$[20,21]$} & $\begin{array}{l}\text { Toothed } \\
\text { ixeridium/Toothed ixeris }\end{array}$ & $\begin{array}{l}\text { Ixeridium dentatum } \\
\text { (Thunb.) Tzvelev }\end{array}$ & $\begin{array}{l}\text { young leaves, } \\
\text { roots }\end{array}$ & $\begin{array}{l}\text { blanched, cooked as } \\
\text { pancakes, salted }\end{array}$ & {$[18,19,58]$} \\
\hline Chinese clover & Medicago denticulate/M. hispida & leaves, stalks & stir-fried & [20] & Young radish & Raphanus sativus $\mathrm{L}$. & $\begin{array}{l}\text { young leaves, } \\
\text { roots }\end{array}$ & salted & {$[18,19]$} \\
\hline $\begin{array}{l}\text { Chinese mustard/Green } \\
\text { mustard/Leaf-mustard } \\
\text { cabbage/Mustard } \\
\text { cabbag/Mustard greens }\end{array}$ & $\begin{array}{l}\text { Brassica juncea/B. juncea var. } \\
\text { rugosa }\end{array}$ & leaves, stalks & salted, pickled, stir-fried & {$[17-21]$} & Korean ginseng sprout & Panax ginseng Meyer & $\begin{array}{l}\text { young leaves, } \\
\text { stems, roots }\end{array}$ & eaten raw & [59] \\
\hline $\begin{array}{l}\text { East Asian } \\
\text { wildparsley/Mitsuba/Honewort }\end{array}$ & $\begin{array}{l}\text { Cryptotaenia canadensis/C. japonica } \\
\text { t Hassk. }\end{array}$ & leaves, stalks & $\begin{array}{l}\text { eaten raw, blanched, } \\
\text { stir-fried, deep fired, } \\
\text { cooked in soups }\end{array}$ & {$[17-20,58]$} & Mioga ginger & $\begin{array}{l}\text { Zingiber mioga (Thunb.) } \\
\text { Roscoe }\end{array}$ & flower buds & $\begin{array}{l}\text { blanched, cooked in } \\
\text { soups, pickled }\end{array}$ & [18] \\
\hline $\begin{array}{l}\text { Horseradish/Japanese } \\
\text { horseradish/Wasabi }\end{array}$ & Eutrema japonica/Wasabia japonica & leaves, stalks & $\begin{array}{l}\text { eaten raw, cooked in soups, } \\
\text { picked, blanched }\end{array}$ & [18] & Banana bud & Musa spp. & flowers & $\begin{array}{l}\text { eaten raw, blanched, } \\
\text { stir-fried, steamed, } \\
\text { cooked in soups, curries }\end{array}$ & [21] \\
\hline $\begin{array}{l}\text { Japanese radish } \\
\text { greens/Korean radish greens }\end{array}$ & Raphanus sativus & leaves, stalks & $\begin{array}{l}\text { dried leaves are cooked in } \\
\text { soups, stir-fried }\end{array}$ & [18] & & & & & \\
\hline
\end{tabular}


Table 4. Fruit, pod and seed vegetables.

\begin{tabular}{|c|c|c|c|c|c|c|c|c|c|}
\hline Vegetable & Scientific Names & Used Parts & Culinary Uses & Reference & Vegetable & Scientific Names & Used Parts & Culinary Uses & References \\
\hline $\begin{array}{l}\text { Angled gourd/Dishcloth } \\
\text { gourd/Vegetable } \\
\text { gourd/Luffa/Sponge gourd }\end{array}$ & Luffa acutangula & fruits & cooked in soups, stir-fried & {$[17,20,21]$} & Spaghetti marrow & $\begin{array}{l}\text { Cucurbita pepo var. } \\
\text { fastigata }\end{array}$ & fruits & steamed, baked, stir-fried & [20] \\
\hline $\begin{array}{l}\text { Balsam-pear/Bitter } \\
\text { gourd/Bitter melon }\end{array}$ & Momordica charantia & fruits & $\begin{array}{l}\text { steamed, stir-fried, cooked in } \\
\text { soups, in stews }\end{array}$ & {$[17,19-21]$} & Baby corn/Miniature corn & Zea mays var. rugosa & $\begin{array}{l}\text { corns with } \\
\text { cobs }\end{array}$ & eaten raw, stir-fried & [21] \\
\hline $\begin{array}{l}\text { Bottle gourd/Edible bottle } \\
\text { gourd/White-flowered gourd }\end{array}$ & $\begin{array}{l}\text { Lagenaria leucantha/L. } \\
\text { siceraria/L. vulgaris/ }\end{array}$ & fruits & $\begin{array}{l}\text { eaten raw, dried, stir-fried, } \\
\text { cooked in soups }\end{array}$ & [17-21] & $\begin{array}{l}\text { Asparagus bean/Cow } \\
\text { pea/Long bean/Yard long } \\
\text { bean/Yard-Long beans }\end{array}$ & $\begin{array}{l}\text { Dolichos } \\
\text { sesquipedalis/Vigna } \\
\text { sesquipedalis/Vigna sinensis } \\
\text { var. sesquipedalis }\end{array}$ & pods & $\begin{array}{l}\text { eaten raw, stir-fried, } \\
\text { cooked in stews }\end{array}$ & {$[17,20,21]$} \\
\hline $\begin{array}{l}\text { Chinese preserving melon/Wax } \\
\text { gourd/Winter gourd }\end{array}$ & $\begin{array}{l}\text { Benincasa cerifera/B. } \\
\text { hispida }\end{array}$ & fruits & $\begin{array}{l}\text { eaten raw, cooked in soups, in } \\
\text { stews, pickled, steamed, } \\
\text { stir-fried }\end{array}$ & {$[17,18,20,21]$} & $\begin{array}{l}\text { Asparagus pea/Goa } \\
\text { beans Winged bean }\end{array}$ & $\begin{array}{l}\text { Psophocarpus } \\
\text { tetragonolobus }\end{array}$ & pods & blanched, stir-fried & {$[17,21]$} \\
\hline Eggplant & Solanum melongena & fruits & $\begin{array}{l}\text { steamed, stir-fried, deep fried, } \\
\text { pan fried, grilled, cooked in } \\
\text { soups, in stews }\end{array}$ & {$[18,21]$} & Edible-podded peas & $\begin{array}{l}\text { Pisum sativum var. } \\
\text { macrocarpon }\end{array}$ & pods & stir-fried & [17] \\
\hline Fiery little bird's-eye chili & Capsicum frutescens & fruits & eaten raw, cooked & [21] & Snow pea/Sugar pea & $\begin{array}{l}\text { Pisum sativum var. } \\
\text { saccaratum }\end{array}$ & pods & blanched, stir-fried & [21] \\
\hline Finger-length chili & $\begin{array}{l}\text { Capsicum annuит cv. } \\
\text { group longum }\end{array}$ & fruits & eaten raw, cooked & [21] & Adzuki bean & $\begin{array}{l}\text { Phaseolus angularis/Vigna } \\
\text { angularis }\end{array}$ & beans & cooked gruel, sweet paste & {$[18,20]$} \\
\hline Hairy melon/Jointed gourd & $\begin{array}{l}\text { Benincasa hispida var. } \\
\text { chieh-gua }\end{array}$ & fruits & $\begin{array}{l}\text { eaten raw, steamed, cooked in } \\
\text { soups, stir-fried }\end{array}$ & [20] & $\begin{array}{l}\text { Borlotti } \\
\text { bean/Red-streaked bean }\end{array}$ & Phaseolus vulgaris cv. & beans & $\begin{array}{l}\text { simmered, cooked in soups, } \\
\text { stews }\end{array}$ & [21] \\
\hline $\begin{array}{l}\text { Japanese pumpkin/Kabocha } \\
\text { squash }\end{array}$ & $\begin{array}{l}\text { Cucurbita maxima/C. } \\
\text { moschata/ some hybrids } \\
\text { between the two }\end{array}$ & fruits & $\begin{array}{l}\text { Steamed, deep fried, cooked } \\
\text { pancakes, porridge }\end{array}$ & {$[18,20]$} & Cowpeas & $\begin{array}{l}\text { Vigna sinensis/ } V \text {. } \\
\text { unguiculate } \mathrm{L} \text {. Walp. }\end{array}$ & beans & $\begin{array}{l}\text { grounded, cooked gruel, } \\
\text { pancakes, jelly }\end{array}$ & {$[17-19]$} \\
\hline $\begin{array}{l}\text { Korean zucchini/Young } \\
\text { pumpkin }\end{array}$ & Cucurbita moschata & fruits & $\begin{array}{l}\text { stir-fried, cooked in soups, } \\
\text { pancakes }\end{array}$ & {$[18,19]$} & Jwinunikong & Rhynchosia nulubilis & beans & cooked, roasted, boiled tea & [18] \\
\hline Long eggplant & $\begin{array}{l}\text { Solanum melongena var. } \\
\text { serpentinum }\end{array}$ & fruits & steamed, stir-fried & {$[17,19]$} & Mungbean & Vigna radiata & beans & $\begin{array}{l}\text { grounded, cooked gruel } \\
\text { and pancakes, jelly }\end{array}$ & {$[18,19]$} \\
\hline Oriental cucumber & Cucumis satious & fruits & eaten raw, salted, pickled & [20] & Soya bean/Soybeans & Glycine hispida/G. max & beans & $\begin{array}{l}\text { grounded, cooked and } \\
\text { made tofu, pastes, sauces, } \\
\text { sweets, side dishes }\end{array}$ & {$[17-20]$} \\
\hline Oriental pickling melon & $\begin{array}{l}\text { Cucumis melo var. } \\
\text { conomon }\end{array}$ & fruits & pickled & [18] & Sword bean & Canavalia ensiformis & beans & $\begin{array}{l}\text { cooked, boiled, steamed, } \\
\text { roasted and boiled tea }\end{array}$ & {$[18,19]$} \\
\hline $\begin{array}{l}\text { Oriental pickling } \\
\text { melon/Pickling melon }\end{array}$ & $\begin{array}{l}\text { Cucumis melo var. } \\
\text { conomon }\end{array}$ & fruits & $\begin{array}{l}\text { Eaten raw, pickled, baked, } \\
\text { stir-fried, boiled, cooked in } \\
\text { soups }\end{array}$ & {$[17,20]$} & Twisted cluster bean & Parkia speciosa & beans & stir-fried & [21] \\
\hline Papaya & Carica papaya & fruits & $\begin{array}{l}\text { eaten raw, simmered, cooked } \\
\text { in soups }\end{array}$ & [21] & Perilla seed & Perilla frutescens & seeds & $\begin{array}{l}\text { roasted and made oil, } \\
\text { roasted or grounded and } \\
\text { used as seasonings }\end{array}$ & [18] \\
\hline Pea eggplant & Solanum torvum & fruits & eaten raw, cooked in curries & [21] & Sesame seeds & Sesame indicum & seeds & $\begin{array}{l}\text { roasted and made oil, } \\
\text { roasted or grounded and } \\
\text { used as seasonings }\end{array}$ & {$[18,20]$} \\
\hline Snake gourd & $\begin{array}{l}\text { Trichosanthes cucumerina } \\
\text { var. anguina }\end{array}$ & fruits & stir-fried, cooked in curries & [21] & $\begin{array}{l}\text { Water caltrop/Water } \\
\text { chestnut }\end{array}$ & $\begin{array}{l}\text { Trapa bicornis/T. bispinosa } \\
\text { T. natans }\end{array}$ & seeds & boiled & {$[17,20,58]$} \\
\hline
\end{tabular}


Table 5. Root vegetables.

\begin{tabular}{|c|c|c|c|c|c|c|c|c|c|}
\hline Vegetable & Scientific Names & Used Parts & Culinary Uses & Reference & Vegetable & Scientific Names & Used Parts & Culinary Uses & Reference \\
\hline $\begin{array}{l}\text { Arrowhead/Chinese } \\
\text { potato/Swamp potato }\end{array}$ & Sagittaria sagittifolia/S. sinensis & tubers & $\begin{array}{l}\text { boiled, fried, stir-fried, } \\
\text { grilled }\end{array}$ & {$[17,20]$} & Gegeol radish & Raphanus sativus & roots, leaves & salted, pickled & [18] \\
\hline Chinese artichoke & $\begin{array}{l}\text { Stachys affinis/S. Sieboldii/S. } \\
\text { tubifera }\end{array}$ & tubers & $\begin{array}{l}\text { eaten raw, blanched, } \\
\text { stir-fried, steamed, } \\
\text { pickled }\end{array}$ & [20] & Japanese turnips & Brassica rapa var. rapifera & roots, leaves & $\begin{array}{l}\text { eaten raw, boiled, stir-fried, } \\
\text { cooked in soups }\end{array}$ & [20] \\
\hline $\begin{array}{l}\text { Chinese potato/Chinese } \\
\text { yam/Cinnamon-vine/East } \\
\text { Asian mountain yam/Yam }\end{array}$ & $\begin{array}{l}\text { Dioscorea batatas/D. japonica } \\
\text { Thunb./D. opposite }\end{array}$ & tubers & $\begin{array}{l}\text { eaten raw, steamed, } \\
\text { cooked gruel and } \\
\text { pancakes }\end{array}$ & {$[17-20,58]$} & Korean radish & Raphanus sativus & roots, leaves & $\begin{array}{l}\text { eaten raw, salted, cooked in } \\
\text { soups, dried }\end{array}$ & {$[18,19]$} \\
\hline Baker's garlic/Rakkyo & Allium chinense & bulbs & pickled & [20] & Small radish & Raphanus sativus L. & roots, leaves & salted & {$[18,19]$} \\
\hline $\begin{array}{l}\text { Chinese taro/Japanese } \\
\text { taro/Taro/ }\end{array}$ & Colocasia esculenta & corms & $\begin{array}{l}\text { cooked in soups, stews, } \\
\text { grilled, steamed, } \\
\text { simmered }\end{array}$ & [17-21] & Balloon-flower & $\begin{array}{l}\text { Platycodon grandifloras (Jacq.) } \\
\text { A.DC. }\end{array}$ & roots & eaten raw, boiled as tea & {$[18,19,58]$} \\
\hline Gonyak & Amorphophallus konjac & corms & $\begin{array}{l}\text { Its starch is cooked and } \\
\text { made jelly }\end{array}$ & [18] & $\begin{array}{l}\text { Bonnet } \\
\text { bellflower/Deodeok/Lance } \\
\text { Asiabell }\end{array}$ & $\begin{array}{l}\text { Codonopsis lanceolate (Siebold } \\
\text { \& Zucc.) Benth. \& Hook.f. ex } \\
\text { Trautv. }\end{array}$ & roots & eaten raw, grilled, salted & {$[18,19,58]$} \\
\hline Water chestnut & $\begin{array}{l}\text { Eleocharis dulcis (Burm.f.) Trin. } \\
\text { ex Hensch./E. tuberosa }\end{array}$ & corms & $\begin{array}{l}\text { eaten raw, boiled, pickled, } \\
\text { grilled }\end{array}$ & {$[20,21,58]$} & Burdock/Great burdock & $\begin{array}{l}\text { Arctium lappa/A. majus/Lappa } \\
\text { major, L. edulis }\end{array}$ & roots & $\begin{array}{l}\text { blanched, pickled, stir-fried, } \\
\text { deep fried, cooked in soups }\end{array}$ & {$[17,19,20]$} \\
\hline Lotus roots & Nelumbo nucifera & rhizomes & $\begin{array}{l}\text { eaten raw, steamed, } \\
\text { stir-fried, pickled }\end{array}$ & {$[17-21]$} & $\begin{array}{l}\text { Horseradish/Japanese } \\
\text { horseradish/Wasabi/Wild } \\
\text { wasabi }\end{array}$ & $\begin{array}{l}\text { Eutrema japonica (Miq.) } \\
\text { Koidz./Wasabia japonica }\end{array}$ & roots & $\begin{array}{l}\text { grated roots are used as } \\
\text { seasoning 'Wasabi' for Sushi }\end{array}$ & {$[18-20,58]$} \\
\hline Daikon & Raphanus sativus longipinnatus & roots, leaves & $\begin{array}{l}\text { pickled, simmered, } \\
\text { cooked in soups, dried }\end{array}$ & {$[17-19,21]$} & & & & & \\
\hline
\end{tabular}

Table 6. Mushrooms.

\begin{tabular}{|c|c|c|c|c|c|c|c|c|c|}
\hline Vegetable & Scientific Names & Used Parts & Culinary Uses & Reference & Vegetable & Scientific Names & Used Parts & Culinary Uses & References \\
\hline Juda's ear & $\begin{array}{l}\text { Auricularia } \\
\text { auricula-judae }\end{array}$ & & stir-fried & [18] & Pine mushroom & Tricholoma matsutake Sing. & & eaten raw, grilled & [18] \\
\hline $\begin{array}{l}\text { Lesser rock tripe/Plated rock } \\
\text { tripe/Smooth rock tripe }\end{array}$ & Manna lichen & & blanched, stir-fried & [18] & Straw Mushroom & Volvariella volvacea & & $\begin{array}{l}\text { stir-fried, cooked in } \\
\text { soups }\end{array}$ & [21] \\
\hline $\begin{array}{l}\text { Oak Mushroom/Shiitake } \\
\text { Mushroom }\end{array}$ & Lentinus edodes & & $\begin{array}{l}\text { stir-fried, cooked in stews, } \\
\text { soups, pancakes }\end{array}$ & {$[18,21]$} & Winter mushroom & Flammulina velutipes Sing. & & $\begin{array}{l}\text { stir-fried, cooked in } \\
\text { stews, soups }\end{array}$ & [18] \\
\hline
\end{tabular}


Due to the limited number of publications and information in Europe, we added some commonly consumed or unique Asian vegetables to the list. Interestingly, some vegetables that had been traditionally gathered in the wild have recently been cultivated to provide more choice for consumers looking for diversity and health benefits. These vegetables are becoming more available in both online and offline markets.

Since vegetables have various common English names in the literature, we use their scientific names to avoid confusion and for better accuracy. The Asian vegetables are categorized under six groups: (i) sprout vegetables (Table 1), (ii) tree vegetables (Table 2), (iii) leaf and stem vegetables (Table 3), (iv) fruit, pod and seed vegetables (Table 4), (v) root vegetables (Table 5), and (vi) mushrooms (Table 6).

For the list of Asian vegetables in Tables 1-6, we gathered information from three published sources in English which introduce Asian vegetables, mainly from China, Japan and Southeast Asia. We also referred to information from published literature in Korean from various South Korean government ministries for more specific data. Additionally, we searched for the popularity of each species on the Internet. If the vegetable name did not appear in offline or online markets or in recipes and comments of internet users, we did not include the vegetable name in the list. Furthermore, we discuss four Asian vegetables and their future potential in the European market.

\section{Four Recommended Asian Vegetables}

Several Asian vegetables that were traditionally gathered wild, harvested from fields or forests, are now sold in online and offline markets in South Korea as 'Ssam' or 'Namul' vegetables. Vegetable-consuming cultures can also be found in other Asian countries. Pickled vegetables are consumed in China as 'Pao Cai' and in Japan as 'Tsukemono' and 'Asazuke'. Additionally, most Southeast Asian countries and China typically consume vegetables by using the stir-fried cooking method. The vegetables are consumed mostly in the forms of 'Kimchi', 'Ssam' and 'Namul' as we explain further below.

'Kimchi' consists of salted and fermented vegetables. It is served as a side dish in almost every meal and can be made with various kinds of vegetables such as Chinese cabbage, radish (Raphanus sativus), cucumber (Cucumis satious), and spring onion (Allium fistulosum). Approximately 300 varieties of 'Kimchi' are estimated to exist in Korea [60]. 'Ssam' consists of wrapping fillings such as cooked meat, rice (Oryza sativa) with fresh leafy vegetables such as lettuce (Lactuca sativa) and perilla leaves (Perilla frutescens). Recently, various new leafy vegetables have been introduced for 'Ssam' and landed successfully in the vegetable market. 'Namul' are seasoned fresh vegetables prepared either blanched or raw as side dishes. Various vegetables, not only cultivated but also gathered wild, are used for 'Namul'.

Below, we present four Asian vegetables. They possess health benefits and exhibit increasing demand in the vegetable market. Additionally, they are easy to cultivate and fit the current trends in the European vegetable market because of their unique taste, higher value, decorative ability and small size. We strongly believe that these crops have the potential to be introduced in the European market in the future.

\subsection{Korean Ginseng Sprouts (Panax Ginseng Meyer)}

Korean ginseng is a perennial plant in the Araliaceae family. Korean ginseng has been used as a medicinal plant in East Asia [61] for more than 2,000 years [62]. Ginsenoside (triterpene glycoside saponin) is the main bioactive ingredient in Korean ginseng and is known to have various pharmacological and physiological benefits [61] such as anti-cancer [63], anti-diabetic [64,65], immunomodulatory [63,66], neuroprotective [63], radioprotective [67], anti-amnestic [63], and anti-stress properties [68,69].

Korean ginseng sprout [59] is a new medicinal vegetable recently introduced in the South Korean vegetable market with demand increasing [59]. Korean ginseng is customarily used only for its roots 
and should be grown for about 5-6 years before harvest [70]. Because of this length of time, it is a high-priced product for consumers (Figure 1). In contrast, Korean ginseng sprout uses all parts of the plant, not only roots, but especially the leaves due to their young tender texture. Leaves contain higher levels of certain types of ginsenosides ( $\mathrm{Rg} 1, \mathrm{Rg} 2+\mathrm{Rh} 1, \mathrm{Rd}$, and $\mathrm{Rg} 3)$ than roots [71]. It can be grown in a hydroponic cultivation system either without soil $[72,73]$ or with nursery soil $[59,74]$ Therefore, ginseng sprouts can be produced all year round without using pesticides in appropriate conditions. It only needs to be grown for $2-8$ weeks after transplanting of one-year-old-ginseng-seedlings [59,72].

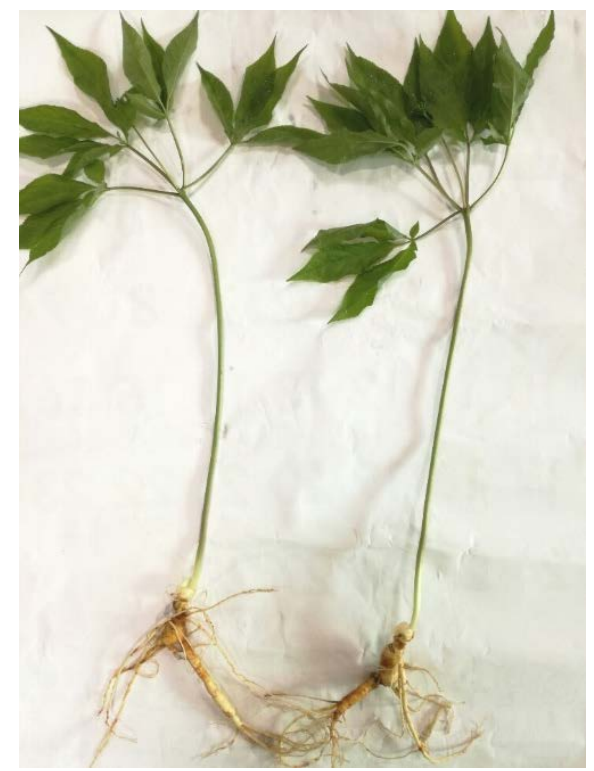

Figure 1. Mountain-cultivated Korean ginseng (Panax ginseng Meyer).

These plants are found in mountainous regions (Gangwon-do, South Korea). Compared to intensive field-cultivated plants, mountain-cultivated ginseng is more expensive. Since wild mountain ginseng is rare and Eastern Asians strongly believe in its health benefits, its value is highly appreciated. Intensively cultivated ginseng needs 5-6 years until harvest, while wild mountain ginseng has a slow growth rate and requires a longer time. Mountain ginseng has a smaller size than intensively cultivated ginseng. Korean ginseng is not usually sold in supermarkets, but in particular markets only for Korean ginseng products or online markets (Source: Hong, personal communication)

Korean ginseng sprouts are a small sized vegetable with a mild, bitter flavor. Soil-less ginseng sprouts can provide health benefits and a pesticide-free alternative for consumers. It can be consumed in a variety of ways, including in salads, milkshakes, deep-fried, sushi, soups, and tea. Furthermore, Korean ginseng sprout can be used in health food supplements and cosmetics.

\subsection{Ssamchoo (Brassica lee ssp. Namai cv. Ssamchoo) and Red Ssamchoo (Brassica koreana Lee var. Redleaf)}

Ssamchoo [18] is a hybrid plant species. This handle-fan-shaped leafy vegetable was developed by aneuploidy crossing Chinese cabbage with regular cabbage as 'Ssam' leafy vegetables [75,76]. Therefore, Ssamchoo combines the advantages of Chinese cabbage and cabbage [76]. Red Ssamchoo (Brassica koreana Lee var. redleaf) is a variety of Green Ssamchoo [76].

According to a leafy vegetables-agricultural technology guide from the Rural Development Association (RDA) South Korea [76], Ssamchoo has all the benefits of Chinese cabbage and cabbage, and a very high fiber content making it easier to digest. Overall contents of calcium, iron, vitamin A and ascorbic acid in Ssamchoo are higher than Chinese cabbage, cabbage and lettuce [76].

Producers can grow Ssamchoo either in soil or hydroponic systems. The method of cultivation is similar to that of lettuce. Ssamchoo can be harvested one month after sowing [76]. However, this vegetable is intolerant to hot and humid weather [76]. 
Ssamchoo has a smaller leaf size (Figure 2) than those of Chinese cabbage and regular cabbage [76]. Red Ssamchoo draws attention due to its red leaf and stem color [76]. Ssamchoo brings together the slight bitterness from Chinese cabbage and nutty sweetness from regular cabbage [76]. This leafy vegetable is lightly aromatic, sweet, juicy, mini-sized, and can be used in salads and soups and may be deep-fried [76].

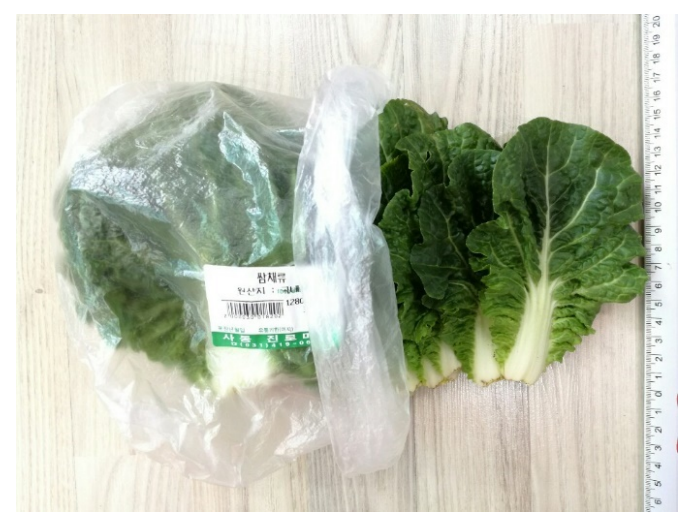

Figure 2. Ssamchoo (Brassica lee ssp. namai cv. Ssamchoo).

Leaves of Ssamchoo can be easily found in open showcases fitted with a cool-mist humidifier in supermarkets. This vegetable is displayed with other various types of fresh leafy vegetables in a 'Ssam-leafy-vegetables-section' of supermarkets. Ssamchoo can be purchased in offline or online stores. Some online stores deliver fresh Ssamchoo leaves within $24 \mathrm{~h}$ after placing an order, even in the early morning of the next day of purchase (Source: Hong, personal collection).

\subsection{Peucedanum Japonicum}

Peucedanum japonicum $[18,19,58]$ is a perennial herb [77] that belongs to the Umbelliferae family and is used as a medicinal plant [78]. This herb is found in China, South Korea, Japan, Taiwan, and the Philippines [79]. Its leaves are consumed traditionally to treat coughs in the Okinawa islands in Japan [78]. Peucedanum japonicum is reported to possess pharmacological benefits such as anti-obesity [78,80,81], anti-oxidant [82], anti-bacterial [83], anti-diabetic [81], tyrosinase inhibition [84], and anti-platelet aggregation [77].

Peucedanum japonicum is traditionally had been foraged from the wild. However, the plant is now cultivated in South Korea for its health benefits (Figure 3). The leaves contain a fragrant aroma, a little bitter and sweet taste. This leafy vegetable can be eaten raw as a salad, blanched, pickled or deep-fried. Peucedanum japonicum can be cultivated in an open field, greenhouse or hydroponic cultivation system $[85,86]$.

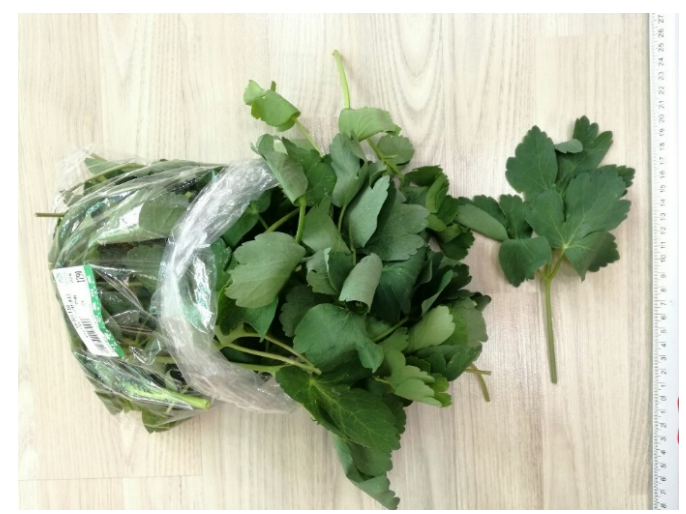

Figure 3. Peucedanum japonicum. 
Young leaves with stems, packed in a plastic bag are sold in 'Namul-leafy vegetable section' of supermarkets. Peucedanum japonicum can be purchased in offline or online stores. Some online stores deliver this fresh leafy vegetable within $24 \mathrm{~h}$ after placing an order, even in the early morning of the day after purchase (Source: Hong, personal collection).

\subsection{Aralia Elata (Miq.) Seem.}

Aralia elata (Miq.) Seem. [18,19,59] is a shrub that is found across Northeastern China, Korea, Japan [87,88] and eastern Russia [89]. In Chinese traditional medicine, its root cortex has been used as a tonic, anti-arthritic and anti-diabetic agent [90]. In the leaves and root cortex, many saponins are isolated and reported to possess an anti-diabetic [90] and a cytoprotective effect [91]. Additionally, a study found specific anti-tumor activity in the extract of the shoots [92].

The young shoots of Aralia elata (Miq.) Seem. have been generally foraged from the wild, but it is cultivated in Japan and South Korea for its expected health benefits (Figure 4). This young shoot has a mildly bitter and sweet taste, fragrant aroma, and a crunchy texture. This shoot vegetable is enough to be an ornament itself in dishes, and thus this young shoot is used as a garnish in Japanese cuisine [93]. In South Korea, the shoots are simply blanched and consumed with a spicy-sour sauce. This aromatic vegetable can also be pickled and deep-fried.

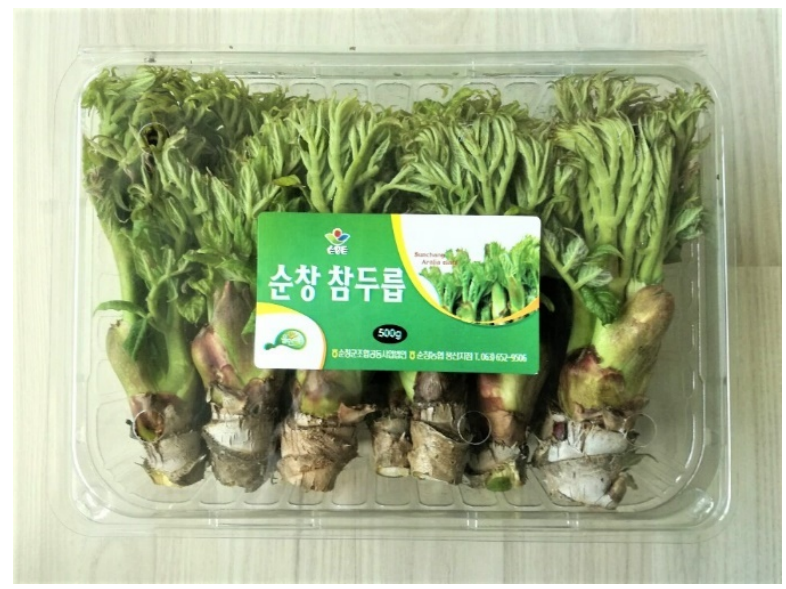

Figure 4. Aralia elata (Miq.) Seem.

Young shoots of Aralia, packed in clear transparent disposable plastic containers can be found in the leafy vegetable section of supermarkets. The shoots are still attached to a small part of woody stems to keep the shoots fresh. Consumers should carefully remove woody stems covered with sharp thorns. Young shoots can be purchased in offline or online stores. Some online stores deliver this fresh young shoots within $24 \mathrm{~h}$ after placing an order, even in the early morning of the day after purchase (Source: Hong, personal collection).

Young shoots of Aralia elata (Miq.) Seem. can be harvested (April-May) in open field cultivation from a whole tree [94,95]. If the shoots are harvested in the greenhouse (February-April), stem cuttings must be prepared [95]. Shoots will develop naturally from the stem cuttings given appropriate humidity [95]. Producers following forcing culture practices need a simple cultivation system such as a double-layer plastic greenhouse, heater, and white greenhouse felt for insulation [95]. Stem cuttings can sprout in immersion cultivation, in which shoots can be harvested after 15 days (30-35 days long) [94]. This method results in harvest at least two weeks earlier than from forcing cultivation [94].

\section{The European Market for Asian Vegetables}

Fresh fruit and vegetable consumption in Europe started to recover slightly in 2014 after suffering declines since 2000 [96]. Daily consumption in the EU-28 stood at $353 \mathrm{~g}$ of fresh fruit and vegetables 
per capita in 2014 [96]. This was split, on average, between $192 \mathrm{~g}$ of fruits and $161 \mathrm{~g}$ of vegetables consumed each day [96]. The consumption of fruits and vegetables in the EU market is expected to increase in the coming decades until 2030 [97]. The main reasons for this phenomenon are increasing interests in a healthy diet and the easier access to fruits and vegetables at new marketplaces, including petrol stations and snack bars [97].

According to Florkowski [98], the market of Asian fruit and vegetables is still small in Europe. However, it is expected to expand across the EU member states, due to increasing consumer interests in diverse, balanced and healthy foods, exchange with Asian people, and contact with the Asian food culture.

When people migrate from one country to another, they have to change their consumption patterns because some vegetables are not available, i.e., the people moving from the Mediterranean region to northern Europe. However, the migrants asking for the vegetables that they were used to eating in their home country find that they cannot be found easily in the new country. In this way, they stimulated the introduction of new vegetables into Europe. An analogous mechanism can be seen during holidays abroad. In foreign countries, people are acquainted with new vegetables. After returning from holiday, they then desire to buy these products in their home country. The introduction of eggplant (Solanum melongena) and zucchini (Cucurbita pepo subsp. pepo convar. giromontiina) to the Dutch [99] and German markets are examples of this.

The small market share of Asian fruits and vegetables in Europe could be further increased through consumer education and safety assurances, as well as marketing campaigns focused on their unique taste, health benefits, ease of preparation, and reasonable price [98]. On the other hand, we believe that the total European fresh vegetable market size will increase over the long term due to strong trends, such as vegetarianism, health and well-being. There is also rising demand from consumers for organic, local, seasonal, fresh, sustainable and new vegetable species and varieties, quality labels, and specific nutrients of vegetables [100]. Thus, increasing demand for locally grown and organic vegetables can lead to the cultivation of Asian vegetables in Europe as a promising business opportunity, especially when these products possess new and additional quality attributes.

Due to stricter European import regulations concerning organic and safety standards of fruits and vegetables, locally-based producers may have comparative advantages in meeting the requirements for supplying fresh Asian vegetables to the European market. For instance, southeastern Asian countries including Malaysia, Thailand and Vietnam which export exotic fruits and vegetables to Europe will need to invest in cultivation practices and laboratory testing for certifications ensuring the hygiene standards of their products [101]. Since most southeastern Asian fruit and vegetable producers are small and live under relatively lower socio-economic conditions, the stricter European regulations are very challenging to meet $[102,103]$.

\subsection{Import from Developing and Asian Countries}

The import of exotic products from developing countries into Europe is continuously increasing [104]. According to a statistical overview from Eurostat on the fruit and vegetable sector in the EU [105], the value of imported fruit and vegetables from developing countries into the EU was approximately 20.1 billion euros in 2017, while the value of exports from the EU to non-Member States was 4.6 billion euros [105]. Fruit imports accounted for $84.7 \%$, while vegetable imports made up $15.3 \%$ of the total value [105]. The reason for this large difference is the relatively higher self-production of vegetables than fruits in Europe [106].

Among Asian countries, China is the leading exporter of vegetables and fruit to the EU. According to data sourced from Eurostat Comext, of total agri-food imports to the EU from China in 2017, fresh, chilled and dried vegetables accounted for $12 \%$ (623 million euros) and prepared vegetables, fruit or nuts accounted for $8 \%$ (416 million euros) [107]. The EU also imports vegetables from South Korea and Japan [108]. Although the European market for fresh fruit and vegetables is highly competitive, this market is large, mature, and has a relatively stable demand [106]. 


\subsection{Exotic and Higher-Value Products}

European consumers have become more interested in exotic and higher-value fruits and vegetables, with the market for these exclusive niche products growing [104]. Niche products and new varieties are expected to continuously emerge, particularly within the high-end market, due to their unique taste and consumer demands [104].

The fruit and vegetable market in Europe focused on unique produces with specific nutritional value is expected to continuously grow. For example, products with specific health benefits such as blueberries, avocados, and pomegranates known commercially as 'superfoods' have become more popular, especially in northwestern Europe [104].

According to CBI research [106], the past five years (2014-2018) have seen the highest growth rates and values from the import of certain fruits from developing countries. For instance, blueberries at $249 \%$ (449 million euro in 2018), avocados at 180\% (1115 million euro) and mangoes at $51 \%$ (778 million euro) [106]. Compared to these exotic fruits, specific vegetables imported from non-EU suppliers are considered to be niche products due to the smaller market in Europe. However, these vegetables showed continuous growth in the European market from 2014 to 2018 with items as chili pepper (Capsicum annuum) at 33\% (48,000 tons in 2018), asparagus (Asparagus officinalis) at 12\% (42,000 tons), tamarinds (Tamarindus indica) at 14\% (41,000 tons), sugar snaps (Pisum sativum var. macrocarpon), snow peas (Pisum sativum var. saccharatum) and other peas (Pisum sativum) at 12\% (30,000 tons) [106].

\subsection{Taste and Emotional Connection}

According to Rabobank [109], the evolving tastes and preferences in developed countries drive the growing value in global trade of fresh fruits and vegetables. The top three drivers are related to the level of customer satisfaction with the quality, fresh appearance, taste, and the size and shape of the products [110]. Taste has recently become a deciding factor for purchase [110]. Additionally, taste preferences are the factors that essentially prevent the consumption of vegetables, especially for younger adults and women in Europe [111]. However, by appealing to the senses - seeing, touching, feeling and smelling - trust and loyalty can be established with customers. Building the emotional connection between consumers and vegetables plays an important role in encouraging customers to make repeated purchases [110]. Even the current Dutch Nationaal Actieplan Groenten en Fruit (National Action-Plan for Vegetables and Fruits) is designed to focus more on emotions and health issues (https://nagf.nl). Besides, sensory appeals of food products, such as taste, texture, quality, smell and appearance, influence consumers to choose an item [112], even if the taste of vegetables does not match the high market price $[100,113]$. Therefore, in-store demonstrations or cooking classes with simple and delicious recipes can provide excellent opportunities for new and unfamiliar Asian vegetables for a successful launch in the European market. With various consumer marketing strategies, the unique tastes and qualities of Asian vegetables can be efficiently introduced to future consumers.

\subsection{Communication with Consumers}

In order to increase the consumption of newly introduced Asian vegetables in the European market, potential European consumers need to aware of these unfamiliar vegetables. For instance, consumers need to know how to select, store, prepare or cook these vegetables. In other words, a successful entry of new Asian vegetables into the European market requires various ways of communication with consumers. Communication channels need to be developed based on the lifestyle and culture of targeted consumers [98]. Websites with easy recipes, such as salads or stir fry, information about health benefits or the use of vegetables can inspire consumers. In addition, social media such as Instagram where people can take, edit, and share photos and videos [114] might contribute to increased demand for new vegetables. For instance, avocado was globally one of the 20 most hash-tagged vegetables on Instagram in 2018 [115]. Avocados had been imported into the European market with the highest growth rate of $180 \%$ for 5 years (2014-2018) and in value in 2018 [106]. 


\section{Asian Vegetables from the Perspective of Climate Change and Cultivation Method}

Trending vegetables in the European market are convenient, healthy, colorful, decorative, organic, local, seasonal [100], mini-sized, and have exceptional nutritional value. Therefore, for a successful entry in the European vegetable market, the introduced Asian vegetables must be tasty, not too exotic, functional, and easy to prepare [98]. For European growers, the new Asian vegetables must be easy to cultivate and should promise a good harvest with high yield. Moreover, these vegetables must be nutritious, clean, fresh, and high-quality to fulfill consumer demands $[100,116]$.

To ensure a holistically sustainable development trajectory, the transformation towards vegetable-rich production and food systems should support climate change adaptation and protection of human and environmental health [117]. Production and the quality of vegetables are being threatened by exposure to an extreme environment, primarily due to climate change-summer heat-waves, heavy rains, drought, possible increasing infestation of weeds, pests, diseases and changes of CO2 concentrations in the air [116-119]. Although traditional outdoor cultivation is cheaper in regards to energy and technology [120], innovative greenhouse cultivation technologies with evaporative cooling, shading, adequate water supply, and drainage systems can secure better vegetable quality $[116,121,122]$.

\section{Difficulties in the Introduction of New Vegetables and Other Ideas of Diversification}

Promoting diverse vegetable supply should be accompanied by different measures-a selection of the best cultivar-genotypes to be grown in the new region, the development of cultivation techniques, avoidance of post-harvest facilities and providing consumers with information about the new ingredients and advice for cocking and preparation. However, the way of introducing new vegetables in the market is not always based on the same pattern. Introduction of kiwi and broccoli in the European market are examples. Even though kiwifruit is originally from China, it was introduced through New Zealand, where the fruit was named after the national "kiwi" bird, to Europe. Broccoli, a native European vegetable with origins in primitive cultivars grown in the Roman Empire, gained its reputation in the European market only after being re-introduced from the USA in the last century.

In many areas of the world, increased consumption of vegetables has been not only because of a growing understanding of the health benefits but also because of increasing availability of various vegetables [39]. Consumers want a continuous supply of vegetables, as well as diversity and novelty, which have become important selling points in modern markets-people are always looking for something new [123]. Introducing new Asian vegetables is just one of the ways to increase the consumption of vegetables in Europe. Introducing colorful vegetables, mini-vegetables, sprout-vegetables, and microgreens are other ways to increase consumption. The new vegetables that have been introduced to the European market include cherry and pear-shaped tomatoes (Solanum lycopersicum), baby-carrots (Daucus carota subsp. sativus), mini-cauliflowers (Brassica oleracea var. botrytis), mini-broccoli (Brassica oleracea var. italica), artichoke hearts (Cynara scolymus), fresh baby leaf curly kale (Brassica oleracea var. sabellica), pickling cucumbers (Cucumis sativus), beans sprouts (Vigna radiata), and different microgreens. The demand for lightly processed, ready-to-eat vegetables is also increasing [39].

Another way to increase vegetable consumption is the time of availability of the products. Year-round vegetable production is possible by using protected cultivation methods. Thus, colorful vegetables could be marketed in the winter period, especially in regions where the vegetable assortment is poor. Even well-known plants in the Mediterranean region, such as eggplant and sweet pepper (Capsicum annuum var. grossum), have been only recently available thanks to greenhouses. Besides, soil-less culture is popularly used because of its advantage in optimizing the root system and efficient control of pathogens without soil fumigation. Therefore, producers can achieve high-quality products as well as higher yields at a reasonable production cost using minimal pesticides [124-126]. Different cultivation methods with porous growing media and water culture systems (floating system, nutrient film technique, and aeroponics) are practiced in commercial greenhouse production [124]. 
In Asia, the indoor production of high-priced and high-quality vegetables is a well-known tradition. Farms use so-called plant factories as highly controlled environments. Figure 5 presents different cultivation steps and plant development stages for Korean ginseng sprouts from a farm in Korea. The vegetable growers of tomatoes, leafy greens, cucumbers, peppers, and others in Europe are progressively using hydroponics primarily because of the high-profit margins and ten times higher yields than traditional cultivation [127]. By using all-environment controlling technologies, producers in Europe can cultivate new Asian vegetables easily and efficiently, meeting food safety standards, only if they are rightly informed about the optimal cultivation conditions of these vegetables. New light processing and packaging technologies will enable fresh vegetables to be presented in a convenient form, e.g., with increased shelf life of pre-cut vegetables while maintaining nutritional value, safety, and freshness [39].

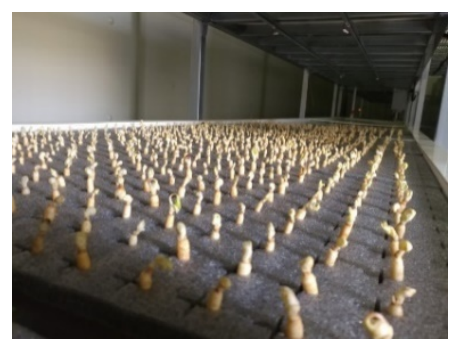

(a)

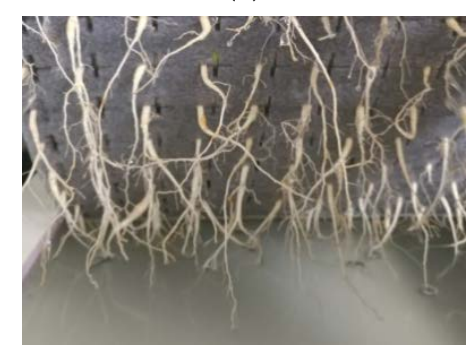

(b)

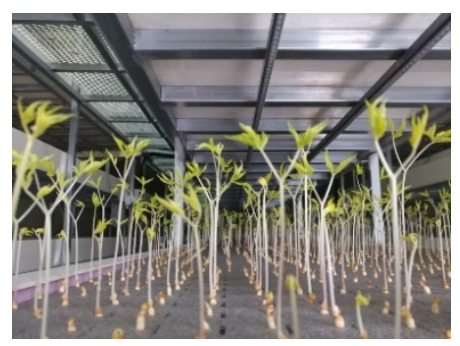

(c)

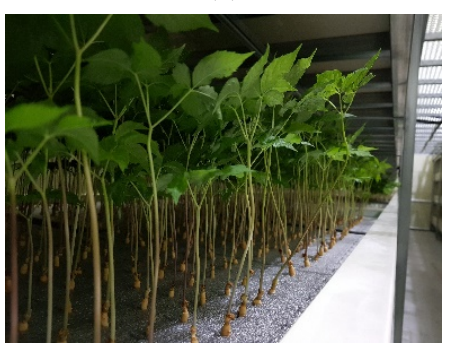

(d)

Figure 5. Cont. 


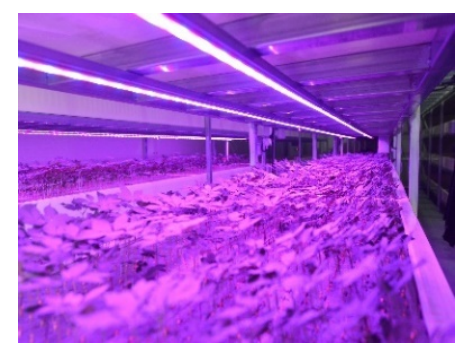

(e)

Figure 5. Plant development stages of Korean ginseng sprouts (Panax ginseng Meyer). Korean ginseng sprouts in a modern plant factory using aquaponics. (a) one-year-old-ginseng-seedlings, freshly transplanted in holes of sponge-hydroponic-floating-rafts; (b) roots of one-year-old-ginseng; (c) roots of about ten-day old cultivated ginseng; (d) at about 21-27 days cultivating ginseng; (e) at about 21-27 days cultivating ginseng seedlings under LED lights. Lighting time: $1-15$ days ( $10 \mathrm{~h}$ exposure/day), after 15 days (13 h exposure/day). Lighting lamps: LED blending ratio (blue:red $=2: 1$ ). The distance between leaves and LED lights about $10 \mathrm{~cm}$ (Source: kindly provided by K. Farm Factory).

\section{Conclusions}

Although the volume of fresh vegetable consumption in the EU is barely increasing, the outlook for the fresh vegetable market is positive. Some of the current trends in the market are the increasing demand for diverse, healthy, and exotic vegetables. In this regard, certain Asian vegetables may have good potential to be introduced and land successfully in the European vegetable market. Here, we discussed the potential of different Asian vegetables for introduction in the European market in the future and shared practical information for the cultivation of four species.

Asian vegetables can be introduced to European consumers not only as fresh vegetables but also as processed products with new and modern ideas, for instance, as Asian vegetable salad bags, as healthy juices, or as cosmetics. In the expanding European health product market, Asian vegetables may play an essential role due to their health benefits and pharmacological properties.

The cultivation of new Asian vegetables within Europe can provide a good opportunity for European vegetable growers to open a new premium niche market with safe, local/seasonal and organic products. For a successful introduction of new Asian vegetables into the European market, appropriate marketing strategies are essential.

Although interest in Asian vegetables is increasing in the European vegetable market, there have been very few studies conducted on Asian vegetables in Europe. From a marketing point of view, the consumer needs and preferences are the key factors leading the production. In complex markets and especially in export-markets, information about consumer needs and wishes does not reach producers all by itself. Thus producers must conduct market research in order to track consumer preferences and to monitor their changes continuously [99]. Further research is required on the Asian vegetable market within Europe and also on the cultivation of potential Asian vegetables under European climatic conditions. Economic, social, and ecological aspects also have to be considered. Further research will provide the basis for all those aspects.

Author Contributions: J.H. and N.S.G. conceptualized and designed the paper, J.H. carried out the literature review and wrote the paper, N.S.G. revised and finalized the paper. All authors have read and agreed to the published version of the manuscript.

Funding: This research received no external funding.

Acknowledgments: We want to thanks to K. Farm Factory, Korea, for providing photos and information about its facility and cultivation conditions.

Conflicts of Interest: The authors declare no conflict of interest. 


\section{References}

1. Suhrcke, M.; McKee, M.; Stuckler, D.; Arce, R.S.; Tsolova, S.; Mortensen, J. The contribution of health to the economy in the European Union. Pub. Health 2006, 120, 994-1001. [CrossRef]

2. Eurostat. Available online: https://ec.europa.eu/eurostat/statistics-explained/index.php/Health_statistics_at_ regional_level (accessed on 9 April 2020).

3. WHO. Available online: http://www.who.int/mediacentre/news/releases/2003/pr20/en/ (accessed on 9 April 2020).

4. Danaei, G.; Ding, E.L.; Mozaffarian, D.; Taylor, B.; Rehm, J.; Murray, C.J.; Ezzati, M. The preventable causes of death in the United States: Comparative risk assessment of dietary, lifestyle, and metabolic risk factors. PLoS Med. 2009, 6, e1000058. [CrossRef]

5. Lim, S.S.; Vos, T.; Flaxman, A.D.; Danaei, G.; Shibuya, K.; Adair-Rohani, H.; Al Mazroa, M.A.; Amann, M.; Anderson, H.R.; Andrews, K.G.; et al. A comparative risk assessment of burden of disease and injury attributable to 67 risk factors and risk factor clusters in 21 regions, 1990-2010: A systematic analysis for the Global Burden of Disease Study 2010. Lancet 2012, 380, 2224-2260. [CrossRef]

6. Lozano, R.; Naghavi, M.; Foreman, K.; Lim, S.; Shibuya, K.; Aboyans, V.; Abraham, J.; Adair, T.; Aggarwal, R.; Ahn, S.Y.; et al. Global and regional mortality from 235 causes of death for 20 age groups in 1990 and 2010: A systematic analysis for the Global Burden of Disease Study 2010. Lancet 2012, 380, 2095-2128. [CrossRef]

7. Visscher, T.L.S.; Seidell, J.C. The public health impact of obesity. Annu. Rev. Pub. Health 2001, 22, 355-375. [CrossRef]

8. OECD/EU. Health at a Glance: Europe 2016: State of Health in the EU Cycle; OECD Publishing: Paris, France, 2016; pp. 55-112, ISBN: 9789264265646 (EPUB), 9789264265592 (PDF); Available online: https:/www.oecd-ilibrary.org/docserver/9789264265592-en.pdf?expires=1586446691\& id=id\&accname=guest\&checksum=C22AA163102617DFEF45BC2DF59DE7D4 (accessed on 9 April 2020).

9. WHO. Available online: https://www.who.int/dietphysicalactivity/childhood_consequences/en/ (accessed on 9 April 2020).

10. WHO. Available online: https://www.who.int/docs/default-source/coronaviruse/situation-reports/20200418sitrep-89-covid-19.pdf?sfvrsn=3643dd38_2 (accessed on 19 April 2020).

11. WHO. Available online: http://www.euro.who.int/en/health-topics/health-emergencies/coronavirus-covid19/weekly-surveillance-report (accessed on 19 April 2020).

12. Chen, N.; Zhou, M.; Dong, X.; Qu, J.; Gong, F.; Han, Y.; Qiu, Y.; Wang, J.; Liu, Y.; Wei, Y.; et al. Epidemiological and clinical characteristics of 99 cases of 2019 novel coronavirus pneumonia in Wuhan, China: A descriptive study. Lancet 2020, 395, 507-513. [CrossRef]

13. Peng, Y.D.; Meng, K.; Guan, H.Q.; Leng, L.; Zhu, R.R.; Wang, B.Y.; He, M.A.; Cheng, L.X.; Huang, K.; Zeng, Q.T. Clinical characteristics and outcomes of 112 cardiovascular disease patients infected by 2019-nCoV. Zhonghua Xin Xue Guan Bing Za Zhi 2020, 48, E004. Available online: https://europepmc.org/article/med/ 32120458 (accessed on 19 April 2020).

14. Saghazadeh, A.; Rezaei, N. Immune-epidemiological parameters of the novel coronavirus-A perspective. Expert Rev. Clin. Immunol. 2020, 16, 1-6. [CrossRef] [PubMed]

15. Maggini, S.; Pierre, A.; Calder, P.C. Immune Function and Micronutrient Requirements Change over the Life Course. Nutrients 2018, 10, 1531. [CrossRef] [PubMed]

16. Gasmi, A.; Noor, S.; Tippairote, T.; Dadar, M.; Menzel, A.; Bjørklund, G. Individual risk management strategy and potential therapeutic options for the COVID-19 pandemic. Clin. Immunol. 2019, 108409. [CrossRef]

17. Chung, H.L.; Ripperton, J.C. Utilization and Composition of Oriental Vegetables in Hawaii; Hawaii Agricultural Experiment Station: Honolulu, HI, USA, 1929; pp. 1-64. Available online: http://hdl.handle.net/10125/14976 (accessed on 9 April 2020).

18. National Institute of Agricultural Sciences-RDA (Rural Development Administration). 9th Revision Korean Food Composition Table II (제9개정판 국가표준 식품성분표II); Functional Food and Nutrition Division-Department of Agro-food Resources-National Institute of Agricultural Sciences-RDA (Rural Development Administration): Wanju, Korea, 2016; ISBN: 978-89-480-4348-8 94520, 978-89-480-4347-2. Available online: http://www.rda.go. kr/download_file/act/bookcafe078.PDF (accessed on 9 April 2020). 
19. Dongbuk Regional Statistics Office-Statistics Korea. Illustrated Crop Book at Each Growth Stages (생 육 단계별 농작물도감); Dongbuk Regional Statistics Office: Daegu, Korea, 2015. Available online: http: //kostat.go.kr/e_book/stepcrop/base/EBook/index.html\#page=10 (accessed on 9 April 2020).

20. Larkcom, J. Oriental Vegetables: The Complete Guide for Garden and Kitchen; John Murray Ltd: London, UK, 1991; ISBN 0719547814.

21. Hutton, W. Handy Pocket Guide to Asian Vegetables; Periplus Editions Ltd.: Hongkong, 2004; ISBN: 0794601944 (ISBN13: 9780794601942).

22. Dixon, G.R. Origins and Diversity of Brassica and its Relatives. In Vegetable Brassicas and Related Crucifers; CABI: Wallingford, UK, 2007; pp. 1-33, ISBN 0-85199-395-8. Available online: https://www.cabi.org/isc/ FullTextPDF/2007/20073006206.pdf (accessed on 9 April 2020).

23. Balvoll, G. Production of Chinese Cabbage in Norway: Problems and Possibilities. J. Veg. Crop Prod. 1995, 1, 3-18. [CrossRef]

24. Bastow, R.; Mylne, J.S.; Lister, C.; Lippman, Z.; Martienssen, R.A.; Dean, C. Vernalization requires epigenetic silencing of FLC by histone methylation. Nature 2004, 427, 164-167. [CrossRef] [PubMed]

25. Chun, C.; Watanabe, A.; Kim, H.H.; Kozai, T. Bolting and Growth of Spinacia oleracea L. can be Altered by Modifying the Photoperiod during Transplant Production. HortScience 2000, 35, 624-626. [CrossRef]

26. Larkcom, J. Oriental Vegetables; Kodansha International Ltd: Tokyo, Japan, 2008; pp. 17-30.

27. Du, Z.; Hou, R.; Zhu, Y.; Li, X.; Zhu, H.; Wang, Z. A random amplified polymorphic DNA (RAPD) molecular marker linked to late-bolting gene in pak-choi (Brassica campestris ssp. chinensis Makino, L.). Afr. J. Biotechnol. 2011, 10, 7962-7968. [CrossRef]

28. Morgan, L. Hydroponic Production of Wasabi \& Japanese Vegetables. In The Best of the Growing Edge International 2000-2005: Select Cream-of-the-Crop Articles for Soilless Growers; Weller, T., Ed.; New Moon Publishing Inc.: Corvallis, OR, USA, 2005; pp. 53-62, ISBN 978-0-944557-05-1.

29. FreshPlaza. Available online: https://www.freshplaza.com/article/9162855/italy-baby-leaf-sector-is-growing (accessed on 28 May 2020).

30. FreshPlaza. Available online: https://www.freshplaza.com/article/2119710/italy-undisputed-leader-of-mixedsalad-sector (accessed on 28 May 2020).

31. Freshplaza. Available online: https://www.freshplaza.com/article/9151954/berlin-start-up-is-dedicated-tocultivating-asian-vegetable-specialties (accessed on 19 June 2020).

32. Freshplaza. Available online: https://www.freshplaza.com/article/9071488/growing-asian-vegetables-inportugal-looks-promising (accessed on 19 June 2020).

33. Agudo, A.; Cabrera, L.; Amiano, P.; Ardanaz, E.; Barricarte, A.; Berenguer, T.; Chirlaque, M.D.; Dorronsoro, M.; Jakszyn, P.; Larrañaga, N.; et al. Fruit and vegetable intakes, dietary antioxidant nutrients, and total mortality in Spanish adults: Findings from the Spanish cohort of the European Prospective Investigation into Cancer and Nutrition (EPIC-Spain). Am. J. Clin. Nutr. 2007, 85, 1634-1642. [CrossRef] [PubMed]

34. Nicklett, E.J.; Semba, R.D.; Xue, Q.L.; Tian, J.; Sun, K.; Cappola, A.R.; Simonsick, E.M.; Ferrucci, L.; Fried, L.P. Fruit and vegetable intake, physical activity, and mortality in older community-dwelling women. J. Am. Geriatr. Soc. 2012, 60, 862-868. [CrossRef]

35. Trichopoulou, A.; Costacou, T.; Bamia, C.; Trichopoulos, D. Adherence to a Mediterranean diet and survival in a Greek population. N. Engl. J. Med. 2003, 348, 2599-2608. [CrossRef]

36. Wang, X.; Ouyang, Y.; Liu, J.; Zhu, M.; Zhao, G.; Bao, W.; Hu, F.B. Fruit and vegetable consumption and mortality from all causes, cardiovascular disease, and cancer: Systematic review and dose-response meta-analysis of prospective cohort studies. BMJ 2014, 349, g4490. [CrossRef]

37. Luzi, L.; Radaelli, M.G. Influenza and obesity: Its odd relationship and the lessons for COVID-19 pandemic. Acta Diabetol. 2020, 1-6. [CrossRef]

38. Shahbaz, M.; Ashraf, M.; Al-Qurainy, F.; Harris, P.J.C. Salt tolerance in selected vegetable crops. Crit. Rev. Plant Sci. 2012, 31, 303-320. [CrossRef]

39. Dias, J.S. World Importance, Marketing and Trading of Vegetables. Acta Hort. 2011, 921, 153-170. [CrossRef]

40. Caton, S.J.; Blundell, P.; Ahern, S.M.; Nekitsing, C.; Olsen, A.; Møller, P.; Hausner, H.; Remy, E.; Nicklaus, S.; Chabanet, C.; et al. Learning to Eat Vegetables in Early Life: The Role of Timing, Age and Individual Eating Traits. PLoS ONE 2014, 9, e97609. [CrossRef] [PubMed] 
41. Anderson, A.S.; Porteous, L.E.G.; Foster, E.; Higgins, C.; Stead, M.; Hetherington, M.; Ha, M.-A.; Adamson, A.J. The impact of a school-based nutrition education intervention on dietary intake and cognitive and attitudinal variables relating to fruits and vegetables. Pub. Health Nutr. 2005, 8, 650-656. [CrossRef]

42. Ransley, J.K.; Greenwood, D.C.; Cade, J.E.; Blenkinsop, S.; Schagen, I.; Teeman, D.; Scott, E.; White, G.; Schagen, $\mathrm{S}$. Does the school fruit and vegetable scheme improve children's diet? A nonrandomised controlled trial. J. Epidemiol. Community Health 2007, 61, 699-703. [CrossRef] [PubMed]

43. Oyebode, O.; Gordon-Dseagu, V.; Walker, A.; Mindell, J.S. Fruit and vegetable consumption and all-cause, cancer and CVD mortality: Analysis of Health Survey for England data. J. Epidemiol. Community Health 2014, 68, 856-862. [CrossRef] [PubMed]

44. Eurostat. Available online: https://ec.europa.eu/eurostat/documents/2995521/7694616/3-14102016-BP-EN. pdf/1234ac94-27fd-4640-b9be-427a42d54881 (accessed on 9 April 2020).

45. Joshipura, K.J.; Hu, F.B.; Manson, J.E.; Stampfer, M.J.; Rimm, E.B.; Speizer, F.E.; Colditz, G.; Ascherio, A.; Rosner, B.; Spiegelman, D.; et al. The effect of fruit and vegetable intake on risk for coronary heart disease. Ann. Intern. Med. 2001, 134, 1106-1114. [CrossRef] [PubMed]

46. Lustig, R.H.; Schmidt, L.A.; Brindis, C.D. The toxic truth about sugar. Nature 2012, 482, 27-29. [CrossRef] [PubMed]

47. Tappy, L.; Lê, K.A.; Tran, C.; Paquot, N. Fructose and metabolic diseases: New findings, new questions. Nutrition 2010, 26, 1044-1049. [CrossRef]

48. Lustig, R.H. Fructose: Metabolic, hedonic, and societal parallels with ethanol. Am. Diet. Assoc. 2010, 110, 1307-1321. [CrossRef]

49. Eurostat. Available online: https://ec.europa.eu/eurostat/statistics-explained/pdfscache/68501.pdf (accessed on 9 April 2020).

50. Kuehnemund, M. Evaluation of the Implementation of the Strategy for Europe on Nutrition, Overweight and Obesity Related Health Issues-Final Report. Available online: https:/ec.europa.eu/health/sites/health/files/ nutrition_physical_activity/docs/pheiac_nutrition_strategy_evaluation_en.pdf (accessed on 9 April 2020).

51. FAO. Available online: http://www.fao.org/3/x0171e/x0171e03.htm (accessed on 28 May 2020).

52. Meldrum, G.; Padulosi, S.; Lochetti, G.; Robitaille, R.; Diulgheroff, S. Issues and prospects for the sustainable use and conservation of cultivated vegetable diversity for more nutrition-sensitive agriculture. Agriculture 2018, 8, 112. [CrossRef]

53. Fritz, D. Starting points for crop research to promote diversification. Acta Hortic. 1989, 242, $193-202$. [CrossRef]

54. OECD. Health at a Glance 2017: OECD Indicators; OECD Publishing: Paris, France, 2017; pp. 76-77, ISBN: 9789264282780 (HTML), 9789264280403 (PDF), 9789264280885 (EPUB). Available online: https://www.oecd-ilibrary.org/docserver/health_glance-2017-en.pdf?expires=1586736825\& $\mathrm{id}=\mathrm{id} \&$ accname$=$ guest\&checksum=43DB9F4E2757464CEA92D340758CE043 (accessed on 9 April 2020).

55. Statista. Available online: https://www.statista.com/statistics/264662/top-producers-of-fresh-vegetablesworldwide (accessed on 9 April 2020).

56. WITS (World Integrated Trade Solution). Available online: https://wits.worldbank.org/CountryProfile/ en/Country/WLD/Year/LTST/TradeFlow/Export/Partner/by-country/Product/06-15_Vegetable (accessed on 9 April 2020).

57. Our World in Data. Available online: https://ourworldindata.org/grapher/average-per-capita-vegetableintake-vs-minimum-recommended-guidelines (accessed on 9 April 2020).

58. Korea National Arboretum. English Names for Korean Native Plants (한반도 자생식물 영어이름 목록집); Korea National Arboretum: Pocheon-si, Korea, 2015; ISBN 978-89-97450-98-5-93480. Available online: http://www.forest.go.kr/kna/special/download/English_Names_for_Korean_Native_Plants.pdf (accessed on 9 April 2020).

59. Jang, I.B.; Yu, J.; Suh, S.J.; Jang, I.B.; Kwon, K.B. Growth and Ginsenoside Content in Different Parts of Ginseng Sprouts Depending on Harvest Time. Korean J. Med. Crop Sci. 2018, 26, 205-213. [CrossRef]

60. Kimchimuseum. Available online: https://www.kimchimuseum.com/kimchi-lab/kimchi-kinds/5529 (accessed on 23 April 2020).

61. Oh, J.Y.; Kim, Y.J.; Jang, M.G.; Joo, S.C.; Kwon, W.S.; Kim, S.Y.; Jung, S.K.; Yang, D.C. Investigation of ginsenosides in different tissues after elicitor treatment in Panax ginseng. J. Ginseng Res. 2014, 38, $270-277$. [CrossRef] [PubMed] 
62. Jung, M.Y.; Jeon, B.S.; Bock, J.Y. Free, esterified, and insoluble-bound phenolic acids in white and red Korean ginsengs (Panax ginseng C. A. Meyer). Food Chem. 2002, 79, 105-111. [CrossRef]

63. Attele, A.S.; Wu, J.A.; Yuan, C.S. Ginseng pharmacology: Multiple constituents and multiple actions. Biochem. Pharmacol. 1999, 58, 1685-1693. [CrossRef]

64. Xie, J.T.; Mehendale, S.R.; Li, X.; Quigg, R.; Wang, X.; Wang, C.Z.; Wu, J.A.; Aung, H.H.; Rue, P.A.; Bell, G.I.; et al. Anti-diabetic effect of ginsenoside Re in ob/ob mice. Biochem. Biophys. Acta 2005, 1740, 319-325. [CrossRef]

65. Shang, W.; Yang, Y.; Zhou, L.; Jiang, B.; Jin, H.; Chen, M. Ginsenoside Rb1 stimulates glucose uptake through insulin-like signaling pathway in 3T3-L1 adipocytes. J. Endocrinol. 2008, 198, 561-569. [CrossRef]

66. Wang, J.; Gao, W.Y.; Zhang, J.; Zuo, B.M.; Zhang, L.M.; Huang, L.Q. Advances in study of ginsenoside biosynthesis pathway in Panax ginseng C. A. Meyer. Acta Physiol. Plant. 2012, 34, 397-403. [CrossRef]

67. Lee, T.K.; Johnke, R.M.; Allison, R.R.; O’Brien, K.F.; Dobbs, L.J., Jr. Radio protective potential of ginseng. Mutagenesis 2005, 20, 237-243. [CrossRef]

68. Saito, H.; Yoshida, Y.; Takagi, K. Effect of Panax ginseng root on exhaustive exercise in mice. Jpn. J. Pharmacol. 1974, 24, 119-127. [CrossRef]

69. Gillis, C.N. Panax ginseng pharmacology: A nitric oxide link? Biochem. Pharmacol. 1997, 54, 1-8. [CrossRef]

70. Soldati, F.; Tanaka, O. Panax ginseng: Relation between age of plant and content of ginsenosides. Planta Med. 1984, 50, 351-352. [CrossRef] [PubMed]

71. Choi, S.Y.; Cho, C.W.; Lee, Y.M.; Kim, S.S.; Lee, S.H.; Kim, K.T. Comparison of Ginsenoside and Phenolic Ingredient Contents in Hydroponically-cultivated Ginseng Leaves, Fruits, and Roots. J. Ginseng Res. 2012, 36, 425-429. [CrossRef] [PubMed]

72. Lee, J.Y.; Yang, H.; Lee, T.K.; Lee, C.H.; Seo, J.W.; Kim, J.-E.; Kim, S.Y.; Park, J.H.Y.; Lee, K.W. A short-term, hydroponic-culture of ginseng results in a significant increase in the anti-oxidative activity and bioactive components. Food Sci. Biotechnol. 2020, 1-6. [CrossRef]

73. Kim, G.S.; Lee, S.E.; Noh, H.J.; Kwon, H.; Lee, S.W.; Kim, S.Y.; Kim, Y.B. Effects of Natural Bioactive Products on the Growth and Ginsenoside Contents of Panax ginseng Cultured in an Aeroponic System. J. Ginseng Res. 2012, 36, 430-441. [CrossRef] [PubMed]

74. Jeong, D.H.; Lee, D.Y.; Jang, I.B.; Yu, J.; Park, K.C.; Lee, E.H.; Kim, Y.J.; Park, H.W. Growth and Ginsenoside Content of One Year Old Ginseng Seedlings in Hydroponic Culture over a Range of Days after Transplanting. Korean J. Med. Crop Sci. 2018, 26, 464-470. [CrossRef]

75. Lee, K.H. Plant Species Ssamchoo and Breeding Method Thereof. U.S. Patent 6,600,092 B2, 29 July 2003. Available online: https://patentimages.storage.googleapis.com/83/67/3a/f2fd2bf0c7a207/US6600092.pdf (accessed on 9 April 2020).

76. RDA (Rural Development Administration). Leafy Vegetables-Agricultural Technology Guide 140 (엽채류-농업기 술길잡이 140(개정 판), 3rd ed.; RDA: Jeonju-si, South Korea, 2017; pp. 142-148, ISBN: 978-89-480-5001-194520, 978-89-480-1772-4(set). Available online: http://lib.rda.go.kr/search/mediaView.do?mets_no=000000299932 (accessed on 9 April 2020).

77. Chen, I.S.; Chang, C.T.; Sheen, W.S.; Teng, C.M.; Tsai, I.L.; Duh, C.Y.; Ko, F.N. Coumarins and antiplatelet aggregation constituents from Formosan Peucedanum japonicum. Phytochemistry 1996, 41, 525-530. [CrossRef]

78. Okabe, T.; Toda, T.; Nukitrangsan, N.; Inafuku, M.; Iwasaki, H.; Oku, H. Peucedanum japonicum Thunb inhibits high-fat diet induced obesity in mice. Phytother. Res. 2011, 25, 870-877. [CrossRef]

79. Chen, C.C.; Agrawal, D.C.; Lee, M.R.; Lee, R.J.; Kuo, C.L.; Wu, C.R.; Tsay, H.S.; Chang, H.C. Influence of LED light spectra on in vitro somatic embryogenesis and LC-MS analysis of chlorogenic acid and rutin in Peucedanum japonicum Thunb.: A medicinal herb. Bot. Stud. 2016, 57, 9. [CrossRef]

80. Nugara, R.N.; Inafuku, M.; Iwasaki, H.; Oku, H. Partially purified Peucedanum japonicum Thunb extracts exert anti-obesity effects in vitro. Nutrition 2014, 30, 575-583. [CrossRef]

81. Nukitrangsan, N.; Okabe, T.; Toda, T.; Inafuku, M.; Iwasaki, H.; Oku, H. Antiobesity activity of Peucednum japonicum Thunb extract in obese diabetic animal model C57 BL/6 J Ham Slc-ob/ob mice. Intern. J. Life Sci. Med. Res. 2012, 2, 28-34. Available online: https://pdfs.semanticscholar.org/a25c/ 4ebb567714c0e702eb25b82cafa7c32f7224.pdf (accessed on 9 April 2020). [CrossRef]

82. Hisamoto, M.; Kikuzaki, H.; Ohigashi, H.; Nakatani, N. Antioxidant compounds from the leaves of Peucedanum japonicum Thunb. J. Agric. Food Chem. 2003, 51, 5255-5261. [CrossRef] [PubMed] 
83. Yang, E.J.; Kim, S.S.; Oh, T.H.; Song, G.; Kim, K.N.; Kim, J.Y.; Lee, N.H.; Hyun, C.G. Peucedenum japonicum and Citrus unshiu essential oils inhibit the growth of antibiotic-resistant skin pathogens. Ann. Microbiol. 2009, 59, 623-628. [CrossRef]

84. Hisamoto, M.; Kikuzaki, H.; Nakatani, N. Constituents of the leaves of Peucedanum japonicum Thunb. and their biological activity. J. Agric. Food Chem. 2004, 52, 445-450. [CrossRef]

85. Lee, G.J.; Heo, J.W.; Jung, C.R.; Kim, H.H.; Yoon, J.B.; Kim, D.E.; Nam, S.Y. Effects of Plant Factory Cultural Systems on Growth, Vitamin C and Amino Acid Contents, and Yield in Hydroponically Grown Peucedanum japonicum. Prot. Hortic. Plant Fact. 2015, 24, 281-286. [CrossRef]

86. Lee, G.J.; Heo, J.W.; Jung, C.R.; Kim, H.H.; Kim, D.E.; Nam, S.Y. Effects of Artificial Light Sources on Growth and Yield of Peucedanum japonicum Hydroponically Grown in Plant Factory. Prot. Hortic. Plant Fact. 2016, 25, 16-23. [CrossRef]

87. Zhang, J.; Wang, H.; Zheng, Q. Cardioprotective effect of Aralia elata polysaccharide on myocardial ischemic reperfusion (IR) injury in rats. Int. J. Biol. Macromol. 2013, 59, 328-332. [CrossRef]

88. Zhang, J.; Wang, H.; Xue, Y.; Zheng, Q. Cardioprotective and antioxidant activities of a polysaccharide from the root bark of Aralia elata (Miq.) Seem. Carbohydr. Polym. 2013, 93, 442-448. [CrossRef]

89. Luo, Y.; Lu, S.; Ai, Q.; Zhou, P.; Qin, M.; Sun, G.; Sun, X. SIRT1/AMPK and Akt/eNOS signaling pathways are involved in endothelial protection of total aralosides of Aralia elata (Miq) Seem against high-fat diet-induced atherosclerosis in ApoE-/-mice. Phytother. Res. 2019, 33, 768-778. [CrossRef]

90. Yoshikawa, M.; Matsuda, H.; Harada, E.; Murakami, T.; Wariishi, N.; Yamahara, J.; Murakami, N.; Elatoside, E. A new hypoglycemic principle from the root cortex of Aralia elata seem.: Structure-related hypoglycemic activity of oleanolic acid glycosides. Chem. Pharm. Bull. 1994, 42, 1354-1356. [CrossRef]

91. Saito, S.; Ebashi, J.; Sumita, S.; Furumoto, T.; Nagamura, Y.; Nishida, K.; Isiguro, I. Comparison of cytoprotective effects of saponins isolated from leaves of Aralia elata Seem. (Araliaceae) with synthesized bisdesmosides of oleanoic acid and hederagenin on carbon tetrachloride-induced hepatic injury. Chem. Pharm. Bull. 1993, 41, 1395-1401. [CrossRef]

92. Tomatsu, M.; Ohnishi-Kameyama, M.; Shibamoto, N. Aralin, a new cytotoxic protein from Aralia elata, inducing apoptosis in human cancer cells. Cancer Lett. 2003, 199, 19-25. [CrossRef]

93. Yoshikawa, M.; Yoshizumi, S.; Ueno, T.; Matsuda, H.; Murakami, T.; Yamahara, J.; Murakami, N. Medicinal foodstuffs. I. Hypoglycemic constituents from a garnish foodstuff "taranome," the young shoot of Aralia elata SEEM.: Elatosides G, H, I, J, and K. Chem. Pharm. Bull. 1995, 43, 1878-1882. [CrossRef] [PubMed]

94. Kim, S.H.; Moon, H.K.; Song, J.H.; Son, S.K.; Lee, J.H. Cultivation Technology for Special Purpose Trees Kalopanax Septemlobu and Aralia Elata (특수수종 음나무 및 두릅나무 재배기술); National Institute of Forest Science: Seoul, South Korea, 2013; pp. 103-169. ISBN 978-89-8176-493-7 93520. Available online: http://know.nifos.go.kr/book/search/DetailView.ax?\&cid=160540 (accessed on 9 April 2020).

95. RDA. Available online: https://www.nongsaro.go.kr/portal/ps/psb/psbk/kidofcomdtyDtl.ps;jsessionid= MFA4MfU8hjAXuTSstduwOhS6BRJbNz6nixtyVGQD21unGS9Y05622tRgnQJCXu5D.nongsaro-web_ servlet_engine1?menuId=PS00067\&kidofcomdtyNo=22534 (accessed on 9 April 2020).

96. Freshfel. Available online: http://freshfel.org/freshfel-consumption-monitor (accessed on 9 April 2020).

97. European Commission. EU Agricultural Outlook for Markets and Income 2018-2030; DG Agriculture and Rural Development: Brussels, Belgium, 2018; pp. 4-73. Available online: https://ec.europa.eu/info/sites/info/ files/food-farming-fisheries/farming/documents/medium-term-outlook-2018-report_en.pdf (accessed on 9 April 2020).

98. Florkowski, W.J. Marketing Asian Fruits and Vegetables in Europe. Acta Hortic. 2008, 804, 39-44. [CrossRef]

99. Van der Plas, M.; Verhaegh, A.P. Marketing of Exotic and Out-of-Season Fresh Fruit and Vegetables in the European Common Market. In Research Report; Wageningen University and Research: Wageningen, The Netherlands, 1986. Available online: https://ibrary.wur.nl/WebQuery/wurpubs/fulltext/264326 (accessed on 28 May 2020).

100. Gruda, N. Assessing the Impact of Environmental Factors on the Quality of Greenhouse Produce. In Achieving Sustainable Greenhouse Cultivation; Marcelis, L., Heuvelink, E., Eds.; Burleigh Dodds Science Publishing Limited: Sawston, UK, 2019; Chapter 16; ISBN 978-1-78676-280-1. [CrossRef]

101. United Nations. Challenges and Opportunities Arising from Private Standards on Food Safety and Environment for Exporters of Fresh Fruit and Vegetables in Asia: Experiences of Malaysia, Thailand and Viet Nam; United Nations 
Publication: New York, NY, USA; Geneva, Switzerland, 2007. Available online: https://unctad.org/en/Docs/ ditcted20076_en.pdf (accessed on 23 April 2020).

102. Faour-Klingbeil, D.; Todd, E.C.D. A Review on the Rising Prevalence of International Standards: Threats or Opportunities for the Agri-Food Produce Sector in Developing Countries, with a Focus on Examples from the MENA Region. Foods 2018, 7, 33. [CrossRef] [PubMed]

103. Grote, U.; Kirchhoff, S. Environmental and Food Safety Standards in the Context of Trade Liberalization: Issues and Options. Discussion Papers on Development Policy No. 39. Available online: https://ageconsearch. umn.edu/record/18725 (accessed on 23 April 2020).

104. CBI Ministry of Foreign Affairs. Available online: https://www.cbi.eu/node/2423/pdf (accessed on 9 April 2020).

105. Eurostat. Available online: https:/ec.europa.eu/eurostat/statistics-explained/index.php/The_fruit_and_ vegetable_sector_in_the_EU_-_a_statistical_overview (accessed on 9 April 2020).

106. CBI Ministry of Foreign Affairs. Available online: https://www.cbi.eu/node/2918/pdf (accessed on 9 April 2020).

107. European Commission. Available online: https:/ec.europa.eu/info/sites/info/files/food-farming-fisheries/ news/documents/agricultural-trade-report_map2018-1_en.pdf (accessed on 9 April 2020).

108. European Commission. Available online: https://ec.europa.eu/info/food-farming-fisheries/trade/agriculturalinternational-trade/bilateral-agreements/asia-and-australasia_en (accessed on 9 April 2020).

109. Rabobank. Available online: https://www.rabobank.com/en/press/search/2016/20160908_fresh_produce_ trade.html (accessed on 9 April 2020).

110. Fruit Logistica. Available online: https://www.fruitlogistica.com/media/fl/fl_images/fl_images_press/6/ pressemitteilungen_3/Fruit_Logistica_Trend_Report_2019.pdf (accessed on 9 April 2020).

111. Pinho, M.G.M.; Mackenbach, J.D.; Charreire, H.; Oppert, J.M.; Bárdos, H.; Glonti, K.; Rutter, H.; Compernolle, S.; De Bourdeaudhuij, I.; Beulens, J.W.J.; et al. Exploring the relationship between perceived barriers to healthy eating and dietary behaviours in European adults. Eur. J. Nutr. 2018, 57, 1761-1770. [CrossRef] [PubMed]

112. Pollard, J.; Kirk, S.F.L.; Cade, J.E. Factors affecting food choice in relation to fruit and vegetable intake: A review. Nutr. Res. Rev. 2002, 15, 373-387. [CrossRef]

113. Gruda, N. Impact of Environmental Factors on Product Quality of Greenhouse Vegetables for Fresh Consumption. Crit. Rev. Plant Sci. 2005, 24, 227-247. [CrossRef]

114. Cambridge Dictionary. Available online: https://ictionary.cambridge.org/dictionary/english/instagram (accessed on 9 April 2020).

115. Sousvidetools.com. Available online: https://www.sousvidetools.com/toolshed/the-most-hashtagged-foodson-instagram (accessed on 23 April 2020).

116. Bisbis, M.B.; Gruda, N.; Blanke, M. Potential impacts of climate change on vegetable production and product quality-A review. J. Clean. Prod. 2018, 170, 1602-1620. [CrossRef]

117. Haddad, L.; Hawkes, C.; Webb, P.; Thomas, S.; Beddington, J.; Waage, J.; Flynn, D. A new global research agenda for food. Nature 2016, 540, 30-32. Available online: https://www.nature.com/news/polopoly_fs/1. 21052!/menu/main/topColumns/topLeftColumn/pdf/540030a.pdf (accessed on 28 May 2020). [CrossRef]

118. Dong, J.; Gruda, N.; Lam, S.K.; Li, X.; Duan, Z. Effects of Elevated CO2 on Nutritional Quality of Vegetables: A Review. Front. Plant Sci. 2018, 9, 924. [CrossRef]

119. Dong, J.; Gruda, N.; Li, X.; Tang, Y.; Zhang, P.; Duan, Z. Sustainable vegetable production under changing climate: The impact of elevated $\mathrm{CO} 2$ on yield of vegetables and the interactions with environments-A review. J. Clean. Prod. 2020, 253, 119920. [CrossRef]

120. Eigenbrod, C.; Gruda, N. Urban vegetable for food security in cities. A review. Agron. Sustain. Dev. 2015, 35, 483-498. [CrossRef]

121. Gruda, N.; Bisbis, M.B.; Tanny, J. Impacts of protected vegetable cultivation on climate change and adaptation strategies for cleaner production-A review. J. Clean. Prod. 2019, 225, 324-339. [CrossRef]

122. Gruda, N.; Bisbis, M.; Tanny, J. Influence of climate change on protected cultivation: Impacts and sustainable adaptation strategies-A review. J. Clean. Prod. 2019, 225, 481-495. [CrossRef]

123. Van Wyk, B.E. The potential of South African plants in the development of new food and beverage products. S. Afr. J. Bot. 2011, 77, 857-868. [CrossRef] 
124. Savvas, D.; Gruda, N. Application of soilless culture technologies in the modern greenhouse industry-A review. Eur. J. Hortic. Sci. 2018, 83, 280-293. [CrossRef]

125. Gruda, N.S. Increasing Sustainability of Growing Media Constituents and Stand-Alone Substrates in Soilless Culture Systems. Agronomy 2019, 9, 298. [CrossRef]

126. Gruda, N. Do soilless culture systems have an influence on product quality of vegetables? J. Appl. Bot. Food Qual. 2009, 82, 141-147. [CrossRef]

127. Businesswire. Available online: https://www.businesswire.com/news/home/20191105005561/en/EuropeHydroponics-Aggregate-Systems-Liquid-Hydroponics-Market (accessed on 9 April 2020).

(C) 2020 by the authors. Licensee MDPI, Basel, Switzerland. This article is an open access article distributed under the terms and conditions of the Creative Commons Attribution (CC BY) license (http://creativecommons.org/licenses/by/4.0/). 